\title{
ENDOMORPHISM ALGEBRAS OF PEAK $I$-SPACES OVER POSETS OF INFINITE PRINJECTIVE TYPE
}

\author{
RÜDIGER GÖBEL AND WARREN MAY
}

\begin{abstract}
We will derive a general result for $R$-categories which allows us to derive the existence of large objects with prescribed endomorphism algebras from the existence of small families. This theorem is based on an earlier result of S. Shelah in which he established the existence of indecomposable abelian groups of any cardinality. We will apply this 'Shelah-elevator' for abelian groups and - which is our main concern - for prescribing endomorphism algebras of peak $I$-spaces which are classified by a recent result of Simson.
\end{abstract}

\section{INTRODUCTION}

In this paper we continue some work started by Gabriel [24] and continued by Brenner [8] and Corner [14] and many others. We will show, for some specific category of $R$-modules which is of infinite representation type, that any $R$-algebra is the endomorphism algebra of "many" of its objects. The category under discussion was investigated recently by Simson [54]. In fact Simson characterized finite prinjective type of the category $P(I, R)$ of peak $I$-spaces over posets $I$ in the case of fields $R$. We will replace the field $R$ by an arbitrary commutative ring $R$ and consider the category $P(I, R)$, where $I$ is of infinite prinjective type. In this case we will realize any $R$-algebra as endomorphism algebra, which illustrates strongly the "wild behaviour" of $P(I, R)$ - even if $I$ is tame but not of finite prinjective type. Using some powerful combinatorial machinery from Shelah $[49,50]$, we are able to describe this wild behaviour in a satisfactory way in terms of endomorphism algebras in case of posets which are of infinite prinjective type.

First we define our objects, which are closely related to classical matrix problems (see Simson [53]).

Let $R$ be any commutative ring with $1 \neq 0$ and $I=(I,<)$ a finite poset. Following [54] we will consider the category $P(I, R)$ of peak $I$-spaces over the commutative ring $R$, whose objects are systems of $R$-modules $\mathbf{M}=\left(M_{i}, \sigma_{i j}\right)$ where $M_{i}$ is an $R$ module, and $\sigma_{i j}$ for all $i \leq j \in I$ is an $R$-homomorphism from $M_{i}$ to $M_{j}$ subject to two conditions:

(1) Compatibility condition: For all $i \leq j \leq k \in I$, we have $\sigma_{i j} \sigma_{j k}=\sigma_{i k}$ and $\sigma_{i i}=i d_{M_{i}}$ is the identity map on $M_{i}$.

Let $\max (I)$ denote the set of maximal elements or peaks of $I$.

If $M_{i}^{\bullet}=\bigoplus_{p \geq i} M_{p}$ denotes the direct sum taken over all $p \in \max (I)$ with $p \geq i$, then we have a canonical R-homomorphism $\sigma_{i}^{\bullet}=\sum_{p \geq i} \sigma_{i p}: M_{i} \rightarrow M_{i}^{\bullet}$.

Secondly we assume

Received by the editors August 24, 1994.

1991 Mathematics Subject Classification. Primary 16G20,16G60. Secondary 20K30. 
(2) Weak injectivity condition: $\sigma_{i}^{\bullet}: M_{i} \rightarrow M_{i}^{\bullet}$ is injective for all $i \in I$.

Particular examples of peak $I$-spaces can be derived from direct sums $M^{\bullet}=$ $\bigoplus_{p \in \max (I)} M_{p}$ and submodules $M_{i} \subseteq M_{i}^{\bullet}$ where $\sigma_{i j}$ is induced by the canonical projection $M_{i}^{\bullet} \rightarrow M_{j}^{\bullet}$.

Finally, morphisms $\varphi: M \rightarrow M^{\prime}$ between objects $\mathbf{M}=\left(M_{i}, \sigma_{i j}\right)$ and $\mathbf{M}^{\prime}=$ $\left(M_{i}^{\prime}, \sigma_{i j}^{\prime}\right)$ are $I$-tuples $\varphi=\left(\varphi_{i}\right)$ of $R$-homomorphisms $\varphi_{i}: M_{i} \rightarrow M_{i}^{\prime}(i \in I)$ compatible with the given $\sigma_{i j}^{\prime}$ 's. Hence the following diagrams commute for all $i \leq j$.

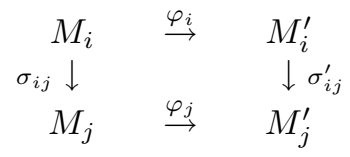

If $I$ has precisely one maximal element $p$ we may ignore the maximal element and consider $J=I \backslash\{p\}$ which is regarded as a subposet of $I$. Then $\sigma_{i}^{\bullet}$ in (2) becomes $\sigma_{i p}$ and all maps $\sigma_{i j}(i<j)$ are likewise injective. Hence $P(I, R)$ is equivalent with the category $J-s p$ of $R$-modules with distinguished submodules partially ordered by $(J,<)$, which was introduced by Gabriel $[24,25,26]$.

This category was investigated over fields $R$ in $[5,8,10,16,17,24,25,26$, $27,32,33,34,35,36,40,41,42,45,46,47,48,51,52,53,54,55]$ (see also [53] for further references). Kleiner $[35,36]$ characterized all posets $J$ with $J-s p$ of finite representation type. This led to the well-known Kleiner-list of 5 minimal counterexamples, the partially ordered sets

\section{$(1,1,1,1),(2,2,2),(1,3,3),,(\mathrm{N}, 4)$ and $(1,2,5)$.}

Then the category $J-s p$ is not of finite representation type if and only if at least one of these orderings can be embedded into $J$.

If $J$ is any antichain of length $n$, then elements in $J-s p$ are called $R_{n}$-modules in Ringel [46, p. 122]. The famous investigation of $\mathbb{Q}_{4}$-modules by Kronecker, who showed that this category is not of finite representation type, is the beginning of this evolution. More recently, on the other hand, Gelfand and Ponomarev [27] and Brenner [10] determined all indecomposable $R_{4}$-modules of finite dimension over fields $R$. However, we can say much more about the structure of modules in $P(I, R)$ if $I$ is not of finite representation type. This can be formulated in terms of algebras realized as endomorphism algebras of peak $I$-spaces over $R$.

The following results - among others - will be special cases of our main theorem, where it is shown that any algebra can be realized in this fashion. Corner [14] proved that any $R$-algebra can be realized over $R_{5}$-Mod (up to certain large cardinals filled in by [21] ). The lower bound $4, R_{4}$-Mod, was reached in [29], and this result was extended to peak $I$-spaces over $R$ with precisely one maximal element in [7] . In order to extend these results to peak $I$-spaces over posets $I$ of infinite prinjective type, we need a classification of posets of finite prinjective type in terms of minimal counterexamples similar to Kleiner's list. Such a classification was established only recently by Simson [54]. Unfortunately we are faced with 114 counterexamples, including 7 infinite families. While Kleiner's 5 examples can be dealt with individually, this is more painful in the present situation. Hence we develop a categorical theorem, which we would like to call "Shelah's elevator". In fact, Shelah [49] developed a combinatorial idea which he used to construct arbitrarily large, indecomposable abelian groups. This special elevator can be enlarged to transport any kind of module structure from one level of cardinality to another. 
This elevator moves modules only upwards to larger cardinal levels! We will also demonstrate its use for other problems in Section 5.

Recall the basic categorical notions from Mitchell [39] .

$W e$ will say that some category $C$ is a pre-module $R$-category if the following conditions hold:

(i) $C$ is an $R$-category:

$\operatorname{Mor}(A, B)$ are $R$-modules and composition is $R$-bilinear for objects $A, B \in C$.

(ii) $C$ is preabelian (hence kernels and cokernels exist).

(iii) Arbitrary direct sums exist in $C$ such that the natural map

$$
\operatorname{Mor}\left(A, \bigoplus_{\kappa} B_{k}\right) \rightarrow \Pi_{\kappa} \operatorname{Mor}\left(A, B_{k}\right)
$$

is always injective.

Then we have the following

Proposition 3. Peak I-spaces over $R$ form a pre-module $R$-category.

A proof is given in Section 6.

In order to formulate Shelah's elevator, we have to define the "ground-level $\omega$ " in terms of an $\omega$-family in a pre-module $R$-category $C$. If $\lambda$ is a cardinal, then $\left\{X_{\alpha} \in C: \alpha \subseteq \lambda\right\}$ will be called a weak $\lambda$-family in $C$, provided it is a $\lambda$ directed system; this means there are morphisms $1_{\alpha \beta}: X_{\alpha} \rightarrow X_{\beta}$ with $1_{\alpha \alpha}=i d_{X_{\alpha}}$, $1_{\alpha \beta} \cdot 1_{\beta \gamma}=1_{\alpha \gamma}$ for all $\alpha \subseteq \beta \subseteq \gamma \subseteq \lambda$, and there are short exact sequences

$$
0 \rightarrow X_{\alpha \cap \beta} \rightarrow X_{\alpha} \oplus X_{\beta} \rightarrow X_{\alpha \cup \beta} \rightarrow 0
$$

with maps induced by the given morphisms.

If such a family is given,

we will say that this family is rigid for some $R$-module $N$ if the following four conditions hold:

(a) $\quad N \otimes X_{\alpha \cap \beta} \rightarrow N \otimes\left(X_{\alpha} \oplus X_{\beta}\right)$ is a semistable kernel, and so are direct sums for various $\alpha$ and $\beta$.

Recall that a kernel is semistable if its pushouts are kernels as well. The notion of semistable kernel is due to Richman and Walker [43].

(b) Any morphism $X_{\alpha} \rightarrow \bigoplus_{\beta \in \lambda} N \otimes X_{\beta}$ factors through a finite direct sum.

(c) If $\varphi: X_{\alpha} \rightarrow N \otimes X_{\alpha}$ and $1_{\emptyset_{\alpha}} \varphi=0$, then $\varphi=0$.

(d)

$$
\operatorname{Mor}\left(X_{\alpha}, N \otimes X_{\beta}\right)=\left\{\begin{array}{c}
N \otimes 1_{\alpha \alpha} \text { if } \alpha=\beta, \\
0 \text { if } \alpha \backslash \beta \text { is infinite. }
\end{array}\right.
$$

Condition (a) reflects that we are working in flat theories, (b) is a special case of "smallness", (c) says that morphisms are already determined on $X_{\emptyset}$ and (d) is a weak form of one of the "classical rigidity conditions", see Fuchs [22, Vol. 2, p. 124].

"Shelah's elevator" is now the following (new)

Theorem $\mathbf{1}^{\prime}$. Let $C$ be a pre-module $R$-category, let $\lambda$ be an infinite cardinal and $A$ be any $R$-algebra with $\leq \lambda$ generators (as algebra). Suppose that we also have a weak $\omega$-family in $C$. Then we can find functors

$$
A \text {-Mod } \rightarrow C \quad\left(\text { denoted } M \rightarrow \mathbf{M}_{\alpha}\right)
$$

for each $\alpha \subseteq \lambda$ such that the following holds: 
If the given weak $\omega$-family in $C$ is rigid for an $A$-module $N$, then

$$
\operatorname{Mor}\left(\mathbf{M}_{\alpha}, \mathbf{N}_{\beta}\right)=\left\{\begin{array}{l}
\operatorname{Hom}_{A}(M, N) \text { if } \alpha \subseteq \beta, \\
0 \text { if } \alpha \nsubseteq \beta,
\end{array}\right.
$$

for all $\alpha, \beta \subseteq \lambda$.

Clearly, if $A=N$ is rigid for a given weak $\omega$-family in $C$, then by the elevator theorem we are led to a "classical rigid" family of $2^{\lambda}$ objects in $C$ with $\operatorname{Mor}\left(N_{\alpha}, N_{\beta}\right)=\delta_{\alpha \beta} \cdot A$. Moreover, the elevator theorem reduces problems on large rigid families to weak rigid $\omega$-families. Its use will be demonstrated in $\S 5$ for classical questions, where we rederive known results. Finally, it was our main intention to apply it to peak $I$-spaces over posets of infinite prinjective type, which is new and will be discussed now.

If $I=(I,<)$ is a finite poset which does not allow peak embeddings of any of the 114 orderings in Simson's list in Section 7, then it follows from Simson [54] that $P(I, R)$ is of finite representation type for any field $R$. Application of Simson [51] or Ringel, Tachikawa [48] shows that all infinite dimensional peak $I$-spaces $M$ on such $I$ are decomposable in $P(I, R)$. In order to realize any $R$-algebra $A$ as an endomorphism algebra $A=$ End $\mathbf{M}$ of an $\mathbf{M}$ in $P(I, R)$ for some $(I,<)$, we must therefore assume that at least one of the orderings in Simson's list can be peakembedded into $(I,<)$, and we may assume without loss of generality that $(I,<)$ is one of them. Just apply the obvious full and faithful induced induction functor $T$ derived from an order monomorphism (see Simson [53, pp. 59-60 and Sect. 17.3]). It remains to find $\omega$-families in these 114 cases and to apply our elevator theorem to derive the desired result.

To reduce the construction of 114 weak $\omega$-families, we adopt a technique from representation theory (see Bernstein, Gelfand and Ponomarev [Usp. Mat. Nauk 28 (1973), 19-33], Ringel [45, pp. 148-155], Simson ${ }^{1}$ or recently Kasjan and Simson [CMS Conf. Proc. 14 (1993), 245-284] and [33] ). This method allows us to deduce infinite representation type of one category from another by what we call "bending arms" (or other order theoretic twists), performed on the poset underlying the first category.

In order to fit this idea into our construction, we have to derive a reduction lemma collecting posets obtained by repeated bending of arms into one case, see Lemma 4 . This way we are able to reduce the 114 cases to 19 , which we then do individually.

We derive the following:

Corollary. For any finite poset $I$, the following are equivalent:

(1) If $R$ is any commutative ring, $\lambda$ is an infinite cardinal such that an $R$ algebra $A$ is generated by $\leq \lambda$ elements, then we can find a $\lambda$-family of peak $I$-spaces $\mathbf{M}_{\alpha}(\alpha \subseteq \lambda)$ in $P(I, R)$ such that for all $\alpha, \beta \subseteq \lambda$

$$
\operatorname{Mor}\left(\mathbf{M}_{\alpha}, \mathbf{M}_{\beta}\right)= \begin{cases}A & \text { if } \alpha \subseteq \beta, \\ 0 & \text { if } \alpha \nsubseteq \beta\end{cases}
$$

(2) The category of peak I-spaces $P(I, R)$ over any field $R$ is not of finite prinjective type.

\footnotetext{
${ }^{1}$ [Fund. Math. 137 (1991), 115-144]
} 
(3) There is a finite poset on the list of Simson's 114 critical posets presented in the appendix of this paper which can be peak-embedded into I.

\section{Categorical PRELiminaries For $C$}

Let $R$ be a commutative ring with $1 \neq 0$ and let $C$ be an additive category such that the following holds:

(C1) $C$ is a left R-category, i.e. the set of morphisms between two objects has a left $R$-module structure such that composition is $R$-bilinear;

(C2) $C$ has kernels and cokernels, i.e. $C$ is preabelian.

In this case we say $0 \rightarrow A \rightarrow B \rightarrow C \rightarrow 0$ is exact if and only if $\alpha=\operatorname{Ker} \beta$ and $\beta=$ Coker $\alpha$, where $\alpha$ is the second map and $\beta$ is the third map of the sequence.

(C3) $C$ has arbitrary direct sums such that whenever all compositions $A \rightarrow$ $\bigoplus_{k \in \kappa} B_{k} \rightarrow B_{l}(l \in \kappa)$ are 0 , then the first morphism is 0 .

We can now define tensor products $M \otimes A \in C$ for $M \in \operatorname{Mod}-R$ and $A \in C$ (cf. Mitchell [39, pp. 142-147]). In brief, choose a free resolution of $M$, say

$$
\bigoplus_{\mu} R \rightarrow \bigoplus_{\nu} R \rightarrow M \rightarrow 0
$$

The homomorphism of the free modules is given by a row finite matrix over $R$. This matrix can be interpreted as a morphism $\bigoplus_{\mu} A \rightarrow \bigoplus_{\nu} A$ since $\operatorname{Mor}(A, A)$ is an $R$-module with distinguished element the identity map. Taking the cokernel, $M \otimes A$ is now defined by the exact sequence

$$
\bigoplus_{\mu} A \rightarrow \bigoplus_{\nu} A \rightarrow M \otimes A \rightarrow 0
$$

Up to isomorphism, $M \otimes A$ is independent of the free resolution chosen. Let $f: M \rightarrow$ $N$ be a homomorphism in Mod- $R$ and $i: A \rightarrow B$ a morphism in $C$. By standard arguments with free resolutions, we obtain a morphism $f \otimes i: M \otimes A \rightarrow N \otimes B$. This tensor bifunctor is right exact and commutes with direct sums in either variable. For a fixed $i: A \rightarrow B$, the map sending $f$ to $f \otimes i$ is a homomorphism $\operatorname{Hom}_{R}(M, N) \rightarrow$ $\operatorname{Mor}(M \otimes A, N \otimes B)$. If we write $\operatorname{Mor}(M \otimes A, N \otimes B)=\operatorname{Hom}_{R}(M, N) \otimes i$, we mean that this homomorphism is bijective.

In the special case $M=R$, we identify $\operatorname{Hom}_{R}(R, N)=N$ and $R \otimes A=A$ and write $\operatorname{Mor}(A, N \otimes B)=N \otimes i$. Using that $\operatorname{Mor}(M \otimes A, N \otimes B)$ is naturally isomorphic to $\operatorname{Hom}_{R}(M, \operatorname{Mor}(A, N \otimes B))$, we easily deduce the

Lemma. If $\operatorname{Mor}(A, N \otimes B)=N \otimes i$, then for all $M \in \operatorname{Mod}-R$,

$$
\operatorname{Mor}(M \otimes A, N \otimes B)=\operatorname{Hom}_{R}(M, N) \otimes i \text {. }
$$

Proof. By hypothesis, we have an isomorphism $N \rightarrow \operatorname{Mor}(A, N \otimes B)$, thus an isomorphism $\operatorname{Hom}_{R}(M, N) \rightarrow \operatorname{Hom}_{R}(M, \operatorname{Mor}(A, N \otimes B))$. If $f \in \operatorname{Hom}_{R}(M, N)$ and

$m \in M$, then the image of $f$ acts on $m$ to give $m f \otimes i$. We also have a composition

$$
\operatorname{Hom}_{R}(M, N) \rightarrow \operatorname{Mor}(M \otimes A, N \otimes B) \rightarrow \operatorname{Hom}_{R}(M, \operatorname{Mor}(A, N \otimes B)),
$$

where the first map is the one under consideration, and the second map is the natural isomorphism. The first map sends $f$ to $f \otimes i$. The image of $f \otimes i$ under the second map acts on $m$ to give the composition

$$
(m \otimes 1) \circ(f \otimes i): A \rightarrow M \otimes A \rightarrow N \otimes B .
$$

But this is just $m f \otimes i$. We conclude that $\operatorname{Hom}_{R}(M, N) \rightarrow \operatorname{Mor}(M \otimes A, N \otimes B)$ is an isomorphism. 
Since $R$ is commutative, the category Mod- $R$ of right $R$-modules can be replaced by the category $R$-Mod of left $R$-modules.

\section{3. $\lambda$-FAMILIES}

The notion of semistable kernels is due to Richman and Walker [43]. A kernel $\varphi: X \rightarrow Y$ is semistable, if for any pushout diagram

$$
\begin{array}{ccc}
X & \stackrel{\varphi}{\rightarrow} & Y \\
\downarrow & & \downarrow \\
X^{\prime} & \stackrel{\varphi^{\prime}}{\rightarrow} & Y^{\prime}
\end{array}
$$

$\varphi^{\prime}$ is a kernel.

A $\lambda$-family $\left\{X_{\alpha}: \alpha \subseteq \lambda\right\}$ in $C$ is a short name for a $\lambda$-directed system of $X_{\alpha} \in C$ and morphisms $1_{\alpha \beta}: X_{\alpha} \rightarrow X_{\beta}$ for $\alpha \subseteq \beta$, such that:

if $\alpha \subseteq \beta \subseteq \gamma$, then $1_{\alpha \gamma}=1_{\alpha \beta} \circ 1_{\beta \nu}$; and $1_{\alpha \alpha}$ is the identity on $X_{\alpha}$.

A weak $\lambda$-family in $C$ is a $\lambda$-family $\left\{X_{\alpha}: \alpha \subseteq \lambda\right\}$ in $C$ such that one has exact sequences

$$
0 \rightarrow X_{\alpha \cap \beta} \rightarrow X_{\alpha} \oplus X_{\beta} \rightarrow X_{\alpha \cup \beta} \rightarrow 0 \text { for all } \alpha \cup \beta \subseteq \lambda,
$$

where the maps are the natural ones induced by $1_{\alpha \cap \beta, \alpha}, 1_{\alpha \cap \beta, \beta}, 1_{\alpha, \alpha \cup \beta}$ and $1_{\beta, \alpha \cup \beta}$.

If $N$ is an R-module, we say the weak $\lambda$-family is rigid for $N$ if the following hold:

(W1) Direct sums of families for various $\alpha, \beta \subseteq \lambda$ of the induced morphism

$$
N \otimes X_{\alpha \cap \beta} \rightarrow N \otimes\left(X_{\alpha} \oplus X_{\beta}\right) \text { are semistable kernels. }
$$

(W2) Any morphism $X_{\alpha} \rightarrow \bigoplus_{\lambda}\left(N \otimes X_{\beta}\right)$ must factor through a finite direct sum;

(W3) If $1_{\emptyset \alpha} \circ \varphi: X_{\emptyset} \rightarrow X_{\alpha} \rightarrow N \otimes X_{\alpha}$ is 0 , then $\varphi=0$; and

$$
\operatorname{Mor}\left(X_{\alpha}, N \otimes X_{\beta}\right)=\left\{\begin{array}{l}
N \otimes 1_{\alpha \alpha} \text { if } \alpha=\beta, \\
0 \text { if } \alpha \backslash \beta \text { is infinite. }
\end{array}\right.
$$

We note that if the weak $\lambda$-family is rigid for $N$, then using (C3) it is rigid for $\bigoplus_{\lambda} N$.

A strong $\lambda$-family in $C$ is a $\lambda$-family $\left\{H_{\alpha}: \alpha \subseteq \lambda\right\}$ in $C$, together with objects $\left\{Q_{i}: i \in \lambda\right\}$ in $C$, and morphisms $H_{\alpha} \rightarrow \bigoplus_{i \in \alpha} Q_{i}$ such that whenever $\alpha \subseteq \beta$, there is a commutative diagram

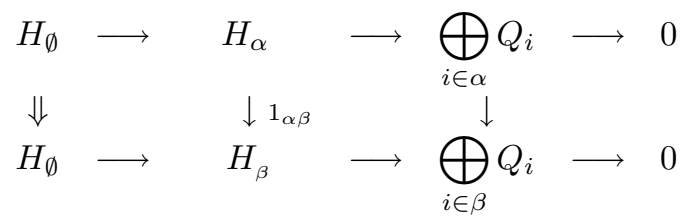

with exact rows, where the left-hand vertical map means equality and the right-hand vertical map is the obvious one.

If $N$ is an $R$-module, we say the strong $\lambda$-family is rigid for $N$ if:

(S1) $N \otimes H_{\alpha} \rightarrow N \otimes H_{\beta}$ is a monomorphism for all $\alpha \subseteq \beta$.

(S2) $\operatorname{Mor}\left(Q_{i}, N \otimes Q_{i}\right)=N \otimes 1_{i}(i \in \lambda)$, where $1_{i}$ denotes the identity on $Q_{i}$; 


$$
\operatorname{Mor}\left(H_{\alpha}, N \otimes H_{\beta}\right)=\left\{\begin{array}{l}
N \otimes 1_{\alpha \beta} \text { if } \alpha \subseteq \beta, \\
0 \quad \text { if } \alpha \nsubseteq \not \beta .
\end{array}\right.
$$

If the family is rigid for every $R$-module, we call it a rigid $\lambda$-family.

Proposition 1. Given a weak $\lambda$-family in $C$, then there exists a strong $\lambda$-family in $C$ which is rigid for every $R$-module $N$ for which the weak $\lambda$-family is rigid.

Proof. Let $\left\{X_{\alpha}: \alpha \subseteq \lambda\right\}$ be a weak $\lambda$-family. Partition $\lambda$ into infinite subsets $\left\{\alpha_{i}: i \in \lambda \cup\{\infty\}\right\}$ and put $Q_{i}=X_{\alpha_{i}}$. Also partition $\alpha_{i}$ into infinite subsets $\alpha_{i 0}$ and $\alpha_{i 1}$.

Now we define $H_{\alpha}$ for $\alpha \subseteq \lambda$ by the commutative diagram

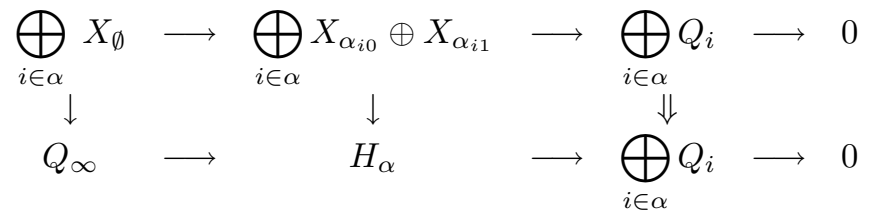

where the upper row is the direct sum of the sequences given by the partitions. The left-hand morphism is the sum morphism of the morphisms coming from the $\lambda$-family, and the left-hand square is a pushout.

Note that $H_{\emptyset}=Q_{\infty}$. We define $H_{\{i\}}=H_{i}$ for $i \in \lambda$. If $\alpha \subseteq \beta$, the natural map of direct sums over $\alpha$ to direct sums over $\beta$ and the fact that $H_{\alpha}$ is a pushout induces $1_{\alpha \beta}: H_{\alpha} \rightarrow H_{\beta}$. It is easy to show that we have a $\lambda$-family and commutative diagrams with exact rows

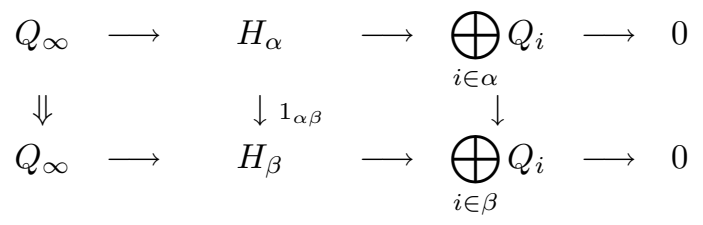

when $\alpha \subseteq \beta$. Thus we have a strong $\lambda$-family.

Now assume the weak $\lambda$-family $\left\{X_{\alpha} ; \alpha \subseteq \lambda\right\}$ is rigid for $N$. Condition (S2) readily follows from (W4). Before verifying (S1) and (S3), we pause to make a useful observation:

$N \otimes_{-}$preserves pushouts.

Therefore, starting with condition (W1) and following the construction of $H_{\alpha}$, we see that we have an exact sequence

$$
0 \rightarrow N \otimes Q_{\infty} \rightarrow N \otimes H_{\alpha} \rightarrow \bigoplus_{i \in \alpha}\left(N \otimes Q_{i}\right) \rightarrow 0 .
$$

Let $\alpha \subseteq \beta$ and $n \in N$. Considering $n \otimes 1_{\alpha \beta}: H_{\alpha} \rightarrow N \otimes H_{\beta}$, we have a commutative diagram

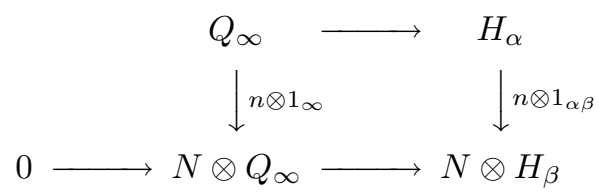

If $n \otimes 1_{\alpha \beta}=0$, then $n \otimes 1_{\infty}=0$; hence $n=0$ by (W4).

Thus we have an injection $N \rightarrow \operatorname{Mor}\left(H_{\alpha}, N \otimes H_{\beta}\right)$. 
To show (S1) and finish showing (S3), let $\varphi: H_{\alpha} \rightarrow N \otimes H_{\beta}$ for arbitrary $\alpha$ and $\beta$. Put $\gamma=\alpha \cup \beta$ and let $k \in \alpha$. We have

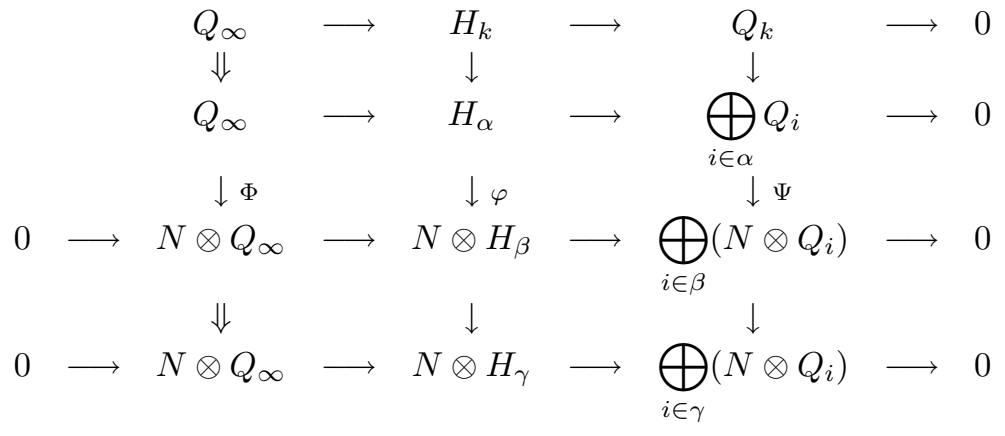

where the upper row does not exist if $\alpha=\emptyset$, and the maps $\Phi, \Psi$ follow from kernel and cokernel arguments since the composition $Q_{\infty} \rightarrow \bigoplus_{i \in \beta}\left(N \otimes Q_{i}\right)$ passing through $\varphi$ must be 0 . To show (S1), in the bottom two rows, the morphisms parallel to $N \otimes H_{\beta} \rightarrow N \otimes H_{\gamma}$ are monomorphisms; thus it is a monomorphism, showing (S1). To show (S3), if $\alpha=\emptyset$, then $H_{\alpha}=Q_{\infty}$ and since the restriction of $\varphi$ to $Q_{\infty} \rightarrow N \otimes Q_{\infty}$ must have form $n \otimes 1_{\infty}$, we have $\varphi=n \otimes 1_{\alpha \beta}$. Thus we may assume $\alpha \neq \emptyset$. The map $\Phi$ is $n \otimes 1_{\infty}$ for some $n \in N$, independent of $k$. Suppose $\alpha \subseteq \beta$. Disregarding the last row and replacing $\varphi$ by $n \otimes 1_{\alpha \beta}$, the left-hand half of the diagram still commutes. Thus the composition $Q_{\infty} \rightarrow H_{\alpha} \rightarrow N \otimes H_{\beta}$ is independent of the replacement. We claim the same is true of $H_{k} \rightarrow H_{\alpha} \rightarrow$ $N \otimes H_{\beta}$ for all $k \in \alpha$. If so, then since $H_{\alpha}$ is a pushout, we will have $\varphi=n \otimes 1_{\alpha \beta}$, as desired. We first claim $\operatorname{Mor}\left(H_{k}, N \otimes Q_{\infty}\right)=0$.

We have a diagram

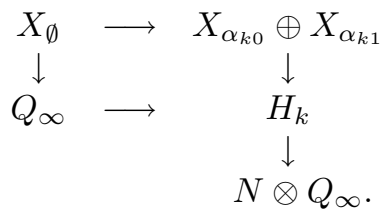

The right vertical composition is 0 by (W4). Therefore the composition $X_{\emptyset} \rightarrow$ $Q_{\infty} \rightarrow N \otimes Q_{\infty}$ is 0 . Thus $Q_{\infty} \rightarrow N \otimes Q_{\infty}$ is 0 by (W3), and since $H_{k}$ is a pushout, we conclude $H_{k} \rightarrow N \otimes Q_{\infty}$ is 0 , proving the claim. Returning to the big diagram without the last row, the right-hand vertical is $n_{k} \otimes 1_{k \beta}$ by (C3) and (W4). Replacing $\varphi$ by $\varphi-n_{k} \otimes 1_{\alpha \beta}$, we deduce that the middle vertical factors through $H_{k} \rightarrow N \otimes Q_{\infty}$. This must be 0 by our claim above. The left-hand rectangle is commutative if we replace the $\Phi$ by $\left(n-n_{k}\right) \otimes 1_{\infty}$. But then it must be 0 ; consequently $n=n_{k}$. Thus the right-hand rectangle is commutative with $\varphi$ replaced by $n \otimes 1_{\alpha \beta}$, and so the composition $H_{k} \rightarrow H_{\alpha} \rightarrow N \otimes H_{\beta}$ remains unchanged and we are done with the case $\alpha \subseteq \beta$.

Now suppose $\alpha \nsubseteq \beta$, choose $k \in \alpha \backslash \beta$, and put the last row back. The middle vertical has form $n_{k} \otimes 1_{k \gamma}$, while the bottom two-thirds has form $n \otimes 1_{\alpha \gamma}$. Since $n_{k} \otimes 1_{k \gamma}=1_{k \alpha} \circ\left(n \otimes 1_{\alpha \gamma}\right)=n \otimes 1_{k \gamma}$, we conclude $n_{k}=n$ by the case of (S3) already proved. The top two-thirds of the right vertical is 0 since $k \notin \beta$; hence $n \otimes 1_{k \gamma}=0$ after cancelling the bottom epimorphism. Thus $n=0$ and hence the bottom twothirds of the middle vertical is 0 . But $N \otimes H_{\beta} \rightarrow N \otimes H_{\gamma}$ is a monomorphism by (S1), and thus $\varphi=0$. 


\section{4. $\lambda$-FAMILIES OF FUNCTORS}

Let $A$ be an R-algebra. A $\lambda$-family of functors from $A$-Mod to $C$ means we are given $\mathbf{M}_{\alpha} \in C$ for all $\alpha \subseteq \lambda$ and $M \in A$-Mod, and $f_{\alpha \beta}: \mathbf{M}_{\alpha} \rightarrow \mathbf{N}_{\beta}$ for all $M, N$ in $A$-Mod, $\alpha \subseteq \beta$ and $f \in \operatorname{Hom}_{A}(M, N)$, such that:

(F1) $\operatorname{Hom}_{A}(M, N) \rightarrow \operatorname{Mor}\left(\mathbf{M}_{\alpha}, \mathbf{N}_{\beta}\right)$ given by $f \rightarrow f_{\alpha \beta}$ is an R-homomorphism;

(F2) $1_{\mathrm{M}}: M \longrightarrow M$ induces the identity $\mathbf{M}_{\alpha} \rightarrow \mathbf{M}_{\alpha}$; and

(F3) if $\alpha \subseteq \beta \subseteq \gamma$ and the diagram

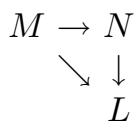

is commutative in A-Mod, then the induced diagram

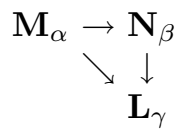

in $C$ is commutative.

Let $N$ be an A-module. We say the $\lambda$-family of functors is rigid for $N$ if for all $M \in A$-Mod,

$$
\operatorname{Mor}\left(\mathbf{M}_{\alpha}, \mathbf{N}_{\beta}\right)=\left\{\begin{array}{l}
\operatorname{Hom}_{A}(M, N) \text { if } \alpha \subseteq \beta, \\
0 \text { if } \alpha \nsubseteq \beta
\end{array}\right.
$$

where "=" means the map in (F1) is bijective.

In the special case of $R_{\kappa}$-Mod, we say that a strong $\lambda$-family $\left\{H_{\alpha}, Q_{i}\right\}$ is $\lambda$-free if for all $k \in \kappa$, the distinguished submodules $H_{\alpha}^{k}$ and $Q_{i}^{k}$ are summands of $H_{\alpha}$ and $Q_{i}$, respectively, such that they and their complements are free $R$-modules of $\operatorname{rank} \lambda$.

Proposition 2. Let $\lambda \geq \kappa \geq \aleph_{o}$ and assume there exists a $\lambda$-free rigid strong $\lambda$ family in $R_{\kappa}$-Mod, and a weak $\kappa$-family in $C$. Let $A$ be an $R$-algebra generated by $\lambda$ elements. Then there exists a $\lambda$-family of functors from $A$-Mod to $C$ which is rigid for every $A$-module $N$ for which the weak $\kappa$-family is rigid.

Proof. Let $\left\{G_{\alpha}: \alpha \subseteq \lambda\right\}$ be a $\lambda$-free rigid strong $\lambda$-family in $R_{\kappa}$-Mod. By Proposition 1, we may assume we have a strong $\kappa$-family $\left\{H_{\gamma}, Q_{i}: \gamma \subseteq \kappa, i \in \kappa\right\}$ in $C$, rigid for the same $N$ as the weak $\kappa$-family. For convenience, we shall assume the $R_{\kappa}$-module structure of $G_{\alpha}$ is given by submodules $G_{\alpha}^{k}$ with $2 \leq k<\kappa$. Choose generators $\left\{a_{i}: i \in \lambda\right\}$ of $A$ and a free basis $\left\{g_{i}, g_{i}^{\prime}: i \in \lambda\right\}$ for $G_{\emptyset}$. Let $M$ be an $A$-module. By (S1), we may regard $M \otimes G_{\emptyset}$ embedded in $M \otimes G_{\alpha}$. Let $M_{\alpha}^{*}$ be the $R$-submodule of $M \otimes G_{\alpha}$ given by $M_{\alpha}^{*}=\bigoplus_{i \in \lambda}\left\{m \otimes g_{i}+a_{i} m \otimes g_{i}^{\prime}: m \in M\right\}$.

We now define $M_{\alpha}=M \otimes G_{\alpha}, M_{\alpha}^{0}=M_{\alpha}, M_{\alpha}^{1}=M_{\alpha}^{*}$ and $M_{\alpha}^{k}=M \otimes G_{\alpha}^{k}$ for $2 \leq k<\kappa$. It is easy to see that the submodules $M_{\alpha}^{k} \quad(k \geq 2)$ give the $R_{\kappa^{-}}$ module structure of $M \otimes G_{\alpha}$. The maps $M_{\alpha}^{k} \rightarrow M_{\alpha}$ and $1_{k \kappa}: H_{k} \rightarrow H_{\kappa}$ induce $\Theta_{M, \alpha}: \bigoplus_{k \in \kappa}\left(M_{\alpha}^{k} \otimes H_{k}\right) \rightarrow M_{\alpha} \otimes H_{\kappa}$. Define $\mathbf{M}_{\alpha} \in C$ such that Coker $\left(\operatorname{Ker} \Theta_{M, \alpha}\right)$ 
is a morphism $\bigoplus_{k \in \kappa}\left(M_{\alpha}^{k} \otimes H_{k}\right) \rightarrow \mathbf{M}_{\alpha}$. Thus we have the commutative diagram

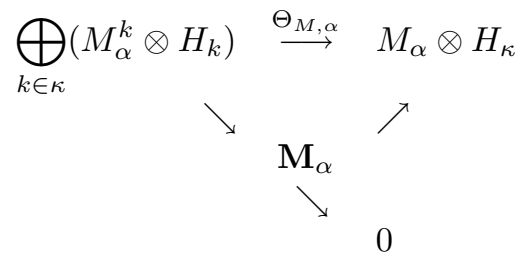

Let $\alpha \subseteq \beta$ and $f \in \operatorname{Hom}_{A}(M, N)$. Then $f \otimes 1_{\alpha \beta}$ is a homomorphism $M_{\alpha} \rightarrow N_{\beta}$ mapping $M_{\alpha}^{k}$ into $N_{\beta}^{k}$ for all $k \in \kappa$. We obtain a commutative square

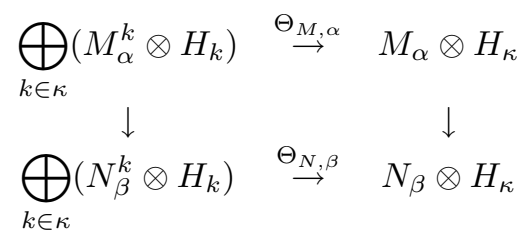

which naturally induces $f_{\alpha \beta}: \mathbf{M}_{\alpha} \rightarrow \mathbf{N}_{\beta}$. One easily verifies that $f \rightarrow f_{\alpha \beta}$ satisfies (F1)-(F3).

Therefore we only need show rigidity.

For the rest of the proof, let $N$ be an $A$-module for which $\left\{H_{\gamma}: \gamma \subseteq \lambda\right\}$ is rigid. By the note after the definition of rigid, the family will also be rigid for $\bigoplus_{\lambda} N$. Suppose that $f_{\alpha \beta}=0$ above. Then the composition $\bigoplus_{\kappa}\left(M_{\beta}^{k} \otimes H_{k}\right) \rightarrow N_{\beta} \otimes H_{\kappa}$ is zero. In particular, taking $k=0$, the map

$$
f \otimes 1_{\alpha \beta} \otimes 1_{0 \kappa}: M \otimes G_{\alpha} \otimes H_{0} \rightarrow N \otimes G_{\beta} \otimes H_{0}
$$

is zero. But $N \otimes G_{\beta} \cong \bigoplus_{\lambda} N$; thus the rigidity assumption (S3) implies $f=0$. Therefore $f \rightarrow f_{\alpha \beta}$ is injective.

Now let $\varphi \in \operatorname{Mor}\left(\mathbf{M}_{\alpha}, \mathbf{N}_{\beta}\right)$ for arbitrary $\alpha$ and $\beta$. Fix $m \in \kappa$ and consider

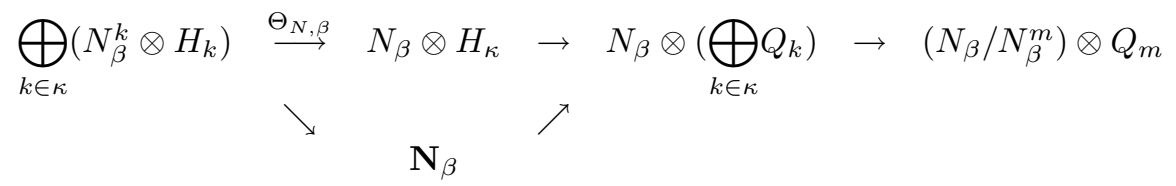

where the last map is induced by the natural map $\pi_{m}: N_{\beta} \rightarrow N_{\beta} / N_{\beta}^{m}$ and the projection $\bigoplus_{k \in \kappa} Q_{k} \rightarrow Q_{m}$. The horizontal composition is clearly 0; thus so is the composition $\mathbf{N}_{\beta} \rightarrow\left(N_{\beta} / N_{\beta}^{m}\right) \otimes Q_{m}$ since the map to $\mathbf{N}_{\beta}$ is an epimorphism. Compose this with $M_{\alpha}^{m} \otimes H_{m} \rightarrow \bigoplus_{k \in \kappa}\left(M_{\alpha}^{k} \otimes H_{k}\right) \rightarrow \mathbf{M}_{\alpha} \rightarrow \mathbf{N}_{\beta}$, again getting zero. Denote the map $\delta_{m}: H_{m} \rightarrow Q_{m}$ given by the strong $\kappa$-family. Regrouping our composition as $M_{\alpha}^{m} \otimes H_{m} \rightarrow N_{\beta} \otimes H_{\kappa} \rightarrow\left(N_{\beta} / N_{\beta}^{m}\right) \otimes Q_{m}$, the left map is $\psi_{m} \otimes 1_{m \kappa}$ for some $\psi_{m}: M_{\alpha}^{m} \rightarrow N_{\beta}$. Thus the composition is $\left(\psi_{m} \circ \pi_{m}\right) \otimes \delta_{m}$. The composition can also be expressed as $M_{\alpha}^{m} \otimes H_{m} \rightarrow M_{\alpha}^{m} \otimes Q_{m} \rightarrow\left(N_{\beta} / N_{\beta}^{m}\right) \otimes Q_{m}$ where the left morphism is an epimorphism since $\delta_{m}$ is. But then the right morphism must be zero, and has the form $\psi_{m} \pi_{m} \otimes 1_{m}$. By the $\lambda$-free assumption, $N_{\beta} / N_{\beta}^{m} \cong \bigoplus_{\lambda} N$, which $\left\{H_{\gamma}: \gamma \subseteq \lambda\right\}$ is rigid for; thus $\psi_{m} \pi_{m}=0$. Consequently $M_{\alpha}^{m} \psi_{m} \subseteq N_{\beta}^{m}$ for all $m \in \kappa$. 
Put $\psi=\psi_{0}: M_{\alpha} \rightarrow N_{\beta}$. We have the commutative square

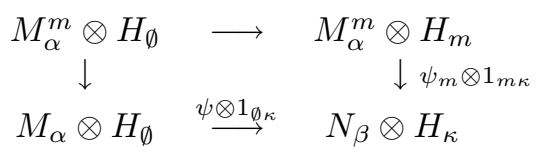

since the top and left maps are equal after composition with $\Theta_{M, \alpha}$, thus are equal after the composition into $\mathbf{M}_{\alpha}$. But this says $\psi_{m} \otimes 1_{\emptyset \kappa}=\left(\psi \mid M_{\alpha}^{m}\right) \otimes 1_{\emptyset \kappa}$. Since $N_{\beta} \cong \bigoplus_{\lambda} N$, by rigidity we get $\psi_{m}=\psi \mid M_{\alpha}^{m}$; thus $M_{\alpha}^{m} \psi \subseteq N_{\beta}^{m}$ for all $m \in \kappa$. In particular $\varphi$ is induced by $\bigoplus_{m \in \kappa}\left(\psi \otimes 1_{m}\right)$. By the rigidity of $\left\{G_{\alpha}: \alpha \subseteq \lambda\right\}$, and noting that $M_{\alpha}^{*} \psi \subseteq N_{\alpha}^{*}$, if $\alpha \subseteq \beta$, then there exists $\psi^{\prime} \in \operatorname{Hom}_{A}(M, N)$ such that $\psi=\psi^{\prime} \otimes 1_{\alpha \beta}$. Hence $\varphi=\left(\psi^{\prime}\right)_{\alpha \beta}$, showing $f \rightarrow f_{\alpha \beta}$ is surjective if $\alpha \subseteq \beta$. If $\alpha \nsubseteq \beta$, then $\psi=0$ by the rigidity, and thus $\varphi=0$.

Remarks. (1) We do not use the exact sequences for $\left\{G_{\alpha} ; \alpha \subseteq \lambda\right\}$ in the proof of Proposition 2, only that the family is $\lambda$-free and satisfies (S1) and (S3) for every $R$-module $N$.

(2) In Proposition 2, suppose that $C=R_{\omega}$-Mod and that $\left\{H_{\gamma} ; \gamma \subseteq \kappa\right\}$ is $\kappa$-free. Take $A=R$ and all $a_{i}=1$. As in [29], $\left\{\mathbf{R}_{\alpha}: \alpha \subseteq \lambda\right\}$ can be verified to be $\lambda$-free. Using that $\left\{G_{\alpha}: \alpha \subseteq \lambda\right\}$ is $\lambda$-free, tensoring the composition $\left(\operatorname{Ker} \Theta_{R, \alpha}\right) \circ \Theta_{R, \alpha}$ with $M$ is exact. Since $M_{\alpha}^{k}=M \otimes R_{\alpha}^{k}$, for all $k$, we obtain $\mathbf{M}_{\alpha}=M \otimes \mathbf{R}_{\alpha}$. Thus $\left\{\mathbf{R}_{\alpha}: \alpha \subseteq \lambda\right\}$ is $\lambda$-free and rigid as in (1).

Theorem 1. Let $\lambda$ be an infinite cardinal and $C$ be an additive category satisfying (C1)-(C3). Assume there exists a weak $\omega$-family in $C$, and let $A$ be an $R$-algebra that can be generated by $\leq \lambda$ elements. Then there exists a $\lambda$-family of functors from $A$-Mod to $C, M \rightarrow \mathbf{M}_{\alpha}(\alpha \subseteq \lambda)$, rigid for every $A$-module $N$ such that the weak $\omega$-family is rigid for $N$. That is, for such $N$ and for $M$ any A-module,

$$
\operatorname{Mor}\left(\mathbf{M}_{\alpha}, \mathbf{N}_{\beta}\right)=\left\{\begin{array}{l}
\operatorname{Hom}_{A}(M, N) \text { if } \alpha \subseteq \beta \subseteq \lambda, \\
0 \quad \text { if } \alpha \nsubseteq \beta .
\end{array}\right.
$$

Proof. By Proposition 2 and Remark 1, it suffices to have a $\lambda$-free $\lambda$-family in $R_{\omega}$-Mod satisfying (S1) and (S3) for every $N$. From references in [29], we obtain such families for various $\lambda$. If $\lambda=\omega$, then Proposition 3.2 [29, p. 37] does it; thus we may assume $\lambda$ uncountable. If $\lambda$ is regular, then Proposition $3.4[29$, p. 37] gives us what we need. If $\lambda$ is singular, then $\kappa=c f \lambda$ is regular; thus we have an appropriate $\kappa$-free $\kappa$-family in $R_{\omega}$-Mod. By Proposition 3.5 in [29, p. 38], there exists a $\lambda$-free rigid $\lambda$-family in $R_{\kappa}$-Mod. Remark 2 now provides an application of Proposition 2 which produces a $\lambda$-free $\lambda$-family in $R_{\omega}$-Mod satisfying (S1) and (S3).

Remark. For appropriate $\lambda$ and $N$, there exist $2^{\lambda}$ non-isomorphic $\mathbf{N}_{\alpha}$, hence $2^{\lambda}$ representations of the algebra $\operatorname{End}_{A} N$.

\section{EXAMPles}

To give examples, we need construct a weak $\omega$-family in $C$ which is rigid for appropriate $A$-modules.

Example 1. The method of primes. 
Let $\lambda$ be an infinite cardinal and $C=\mathbf{Z}$-Mod be the class of abelian groups. Then there exists a $\lambda$-family of functors from $C$ to $C$ which is rigid for $C$. In particular, there exist abelian groups $G_{\alpha}(\alpha \subseteq \lambda)$ such that

$$
\operatorname{Mor}\left(G_{\alpha}, G_{\beta}\right)=\left\{\begin{array}{l}
\mathbf{Z} \text { if } \alpha \subseteq \beta, \\
0 \text { if } \alpha \nsubseteq \beta
\end{array}\right.
$$

Proof. Let $p_{0}, p_{1}, \ldots$ be an enumeration of the prime numbers. For $\alpha \subseteq \omega$, define $X_{\alpha}=\left\{q=\frac{r}{s} \in \mathbf{Q}: s\right.$ is a product of distinct $\left.p_{i}, i \in \alpha\right\}$. Then $X_{\alpha}+X_{\beta}=X_{\alpha \cup \beta}$, $X_{\alpha} \cap X_{\beta}=X_{\alpha \cap \beta}$; thus a pushout construction leads to

$$
0 \longrightarrow X_{\alpha \cap \beta} \longrightarrow X_{\alpha} \oplus X_{\beta} \longrightarrow X_{\alpha \cup \beta} \longrightarrow 0 .
$$

Conditions (W1)-(W4) are all easy to verify for $N=\mathbf{Z}$.

Example 2. The method of transcendentals.

Let $R$ be a commutative ring with $1 \neq 0$ and $S$ be a multiplicative system in $R$ such that $R$ is $S$-reduced and $S$-torsion-free. Let $\lambda$ be an infinite cardinal and $C=R$-Mod. Suppose A is an $R$-algebra generated by $\leq \lambda$ elements. Let $N$ be an $A$-module such that it is $S$-reduced and $S$-torsion-free and the $S$-adic closure of $\mathrm{R}$ has infinitely many algebraically independent elements over $\mathrm{N}$. Then there exists a $\lambda$-family of functors from $A$-Mod to $R$-Mod which is rigid for $N$. For every $A$-module $M$ we have

$$
\operatorname{Mor}\left(\mathbf{M}_{\alpha}, \mathbf{N}_{\beta}\right)=\left\{\begin{array}{l}
\operatorname{Hom}_{A}(M, N) \text { if } \alpha \subseteq \beta, \\
0 \text { if } \alpha \nsubseteq \beta .
\end{array}\right.
$$

In particular, if $M=N=A$, then End $\mathbf{A}_{\alpha}=A$.

Proof. Let $t_{0}, t_{1}, \ldots$ be elements of the $S$-adic completion $\hat{R}$ of $R$ which are algebraically independent over $N$. For $\alpha \subseteq \omega$, define $X_{\alpha}=\left(R+\sum_{i \in \alpha} R t_{i}\right)_{*}$, where the purification is $S$-purification in $\hat{R}$. Since $\hat{R} / R$ is $S$-torsion-free and $S$-divisible, it follows that $X_{\alpha}+X_{\beta}=X_{\alpha \cup \beta}$ and $X_{\alpha} \cap X_{\beta}=X_{\alpha \cap \beta}$. Thus we have the necessary exact sequences provided by pushouts.

Since $X_{\alpha} \subseteq \hat{R}$, we have a natural map $\chi: N \otimes X_{\alpha} \rightarrow \hat{N}$ such that $(n \otimes x) \chi=n x$. We claim that $\chi$ is injective and identifies $N \otimes X_{\alpha}$ with a pure dense submodule of $\hat{N}$. Consider

$$
\sigma \circ \rho \circ \chi: N \otimes R \rightarrow N \otimes\left(R+\sum_{i \in \alpha} R t_{i}\right) \rightarrow N \otimes X_{\alpha} \rightarrow \hat{N} .
$$

The map $\sigma$ is injective since $R$ is a summand, and $\rho \chi$ is injective since the $\left\{t_{i}: i \in\right.$ $\alpha$ \} are independent over $N$. We have an exact sequence

$$
0 \longrightarrow R \longrightarrow X_{\alpha} \longrightarrow X_{\alpha} / R \longrightarrow 0
$$

with $X_{\alpha} / R$ torsion-free, divisible, which induces

$$
0 \longrightarrow N \otimes R \longrightarrow N \otimes X_{\alpha} \longrightarrow N \otimes X_{\alpha} / R \longrightarrow 0
$$

with $N \otimes X_{\alpha} / R$ torsion-free and divisible. Thus $N \otimes X_{\alpha}$ is torsion-free. This, together with the facts that the image of $B$ is $S$-full in $N \otimes X_{\alpha}$, and $\rho \chi$ is injective, yields $\chi$ injective. The image of $\chi$ is pure dense since both $\hat{N} / N$ and

$$
\left(N \otimes X_{\alpha}\right) /(N \otimes R)
$$

are torsion-free divisible. 
Now (W1) follows easily since $\chi$ is injective and all kernels are semistable in $C$. Conditions (W2)-(W3) follow since $R$ is dense in $X_{\alpha}$. By extending homomorphisms to $\hat{R}$-homomorphisms on completions, standard arguments show (W4), using that the $\left\{t_{i} ; i \in \alpha\right\}$ are algebraically independent over $N$.

Example 3. The method of heights.

Let $R$ be a principal ideal domain, $\lambda$ be an infinite cardinal and $C$ be the category of valuated $R$-modules. Let $A$ be a reduced torsion-free $R$-algebra generated by $\lambda$ elements. Then there exists a $\lambda$-family of functors from $A$-Mod to $C$ rigid for every reduced torsion-free $A$-module. That is, for all $A$-modules $M$ and $N$ with $N$ reduced and torsion-free as an $R$-module,

$$
\operatorname{Mor}\left(\mathbf{M}_{\alpha}, \mathbf{N}_{\beta}\right)=\left\{\begin{array}{l}
\operatorname{Hom}_{A}(M, N) \text { if } \alpha \subseteq \beta, \\
0 \text { if } \alpha \nsubseteq \beta .
\end{array}\right.
$$

In particular, End $\mathbf{A}_{\alpha}=A$.

Proof. From [37], take the partition $\omega=\bigcup_{k \in \omega} I_{k}$, and the functions $g$ and $h$ from $\omega$ to $\omega$. For $\alpha \subseteq \omega$ and $N$ a torsion-free $R$-module, define $N_{\alpha} \in C$ as follows. As a module, $N_{\alpha}=N$. If $x \in N_{\alpha}$ and $p$ is a prime, we define the $p$-valuation $v_{p}$ by

$$
\begin{array}{ll}
v_{p}(x)=g(k) & \text { if }|x|_{p} \in I_{k}, k \notin \alpha, \\
v_{p}(x)=h(k) & \text { if }|x|_{p} \in I_{k}, k \in \alpha, \\
v_{p}(x)=\infty & \text { if }|x|_{p}=\infty .
\end{array}
$$

Then $N_{\alpha}$ becomes a valuated $R$-module. It is clear we have a $\lambda$-family since if $\alpha \subseteq \beta$, the identity map on $N$ is a morphism $N_{\alpha} \rightarrow N_{\beta}$. For arbitrary $\alpha$ and $\beta$, the pushout sequence $0 \rightarrow N_{\alpha \cap \beta} \rightarrow N_{\alpha} \oplus N_{\beta} \rightarrow N_{\alpha \cup \beta} \rightarrow 0$ is exact in $C$ since the left map takes the minimum of two values, and the right map takes the maximum. We note that the embedding is nice. Finally, we note that the standard isomorphism $N \otimes R \rightarrow N$ in $R$-Mod induces an isomorphism $N \otimes R_{\alpha} \rightarrow N_{\alpha}$ in $C$. For our weak $\omega$ family, we take $\left\{R_{\alpha} ; \alpha \subseteq \omega\right\}$. Conditions (W1)-(W3) are immediate, (W1) since the embedding is nice. The $\operatorname{Mor}\left(X_{\alpha}, N \otimes X_{\alpha}\right)=N$ part of (W4) is also straightforward. A much simpler version of the proof in [37] shows that $\operatorname{Mor}\left(X_{\alpha}, N \otimes X_{\beta}\right)=0$ if $\alpha \backslash \beta$ is infinite.

\section{Application tO PEAK $I$-SPACES}

Let $I$ be a poset, and recall the definition of "peaks" in $I$, which are the elements in $\max I=\{p \in I: j>p$ for no $j \in I\}$. We assume that for all $i \in I$, the set $I_{i}=\{p \in \max I: p \geq i\}$ is finite. Let $\mathbf{M}=\left(M_{i}, \sigma_{i j}\right)$ be such that $M_{i}$ is an $R$-module for all $i \in I$, and $\sigma_{i j}$ is the an $R$-homomorphism from $M_{i}$ to $M_{j}$ for all $i \leq j$ such that $\sigma_{i i}$ is the identity on $M_{i}$, and $\sigma_{i j} \circ \sigma_{j k}=\sigma_{i k}$ for all $i \leq j \leq k$. If $\mathbf{N}=\left(N_{i}, \tau_{i j}\right)$, then a morphism $\varphi: \mathbf{M} \rightarrow \mathbf{N}$ will be $\varphi=\left(\varphi_{i}\right)$ where $\varphi_{i}: M_{i} \rightarrow N_{i}$ is an $R$-homomorphism and for all $i \leq j \in I$ we have $\varphi_{i} \tau_{i j}=\sigma_{i j} \varphi_{j}$. Define $M^{\bullet}=\bigoplus_{p \in \max I} M_{p}$ and for all $i \in I$, let $M_{i}^{\bullet}=\bigoplus_{p \in I_{i}} M_{p}$. Regard $M_{i}^{\bullet}$ as embedded in $M^{\bullet}$.

Following [54], we say that $\mathbf{M}=\left(M_{i}, \sigma_{i j}\right)$ is a peak $I$-space over $R$ if for all $i \in I$, the map $\sigma_{i}^{\bullet}: M_{i} \rightarrow M_{i}^{\bullet} \subseteq M^{\bullet}$ induced by $\left\{\sigma_{i p}: p \in I_{i}\right\}$ is injective. Thus we may construct particular peak $I$-spaces by regarding all $M_{i}$ as submodules of $M^{\bullet}$ and the maps $\sigma_{i j}$ as induced by the natural projections $M_{i}^{\bullet} \rightarrow M_{j}^{\bullet}$. In this section $C=P(I, R)$ will denote the category of peak $I$-spaces over $R$ (cf. Section 1 ). 
Example. Let $\kappa$ be a cardinal, and $I=\kappa \cup\{p\}$, where $p$ is not an ordinal and the partial order on $I$ is $i<p$ for all $i \in \kappa$. Then $C$ is equivalent to the category of $R_{\kappa}$-modules.

$C$ is clearly an additive category. We verify that it has properties $(\mathrm{C} 1)-(\mathrm{C} 3)$. (C1) is immediate. For (C2) let $\varphi: \mathbf{M} \rightarrow \mathbf{N}$ be a morphism, where $\mathbf{M}=\left(M_{i}, \sigma_{i j}\right)$, $\mathbf{N}=\left(N_{i}, \tau_{i j}\right)$. For every $i \in I$, let $K_{i}=\operatorname{Ker}\left(\varphi_{i}: M_{i} \rightarrow N_{i}\right)$. Then $\sigma_{i j} \mid K_{i}$ maps $K_{i}$ into $K_{j}$. By property of kernels in $R$-Mod, $\mathbf{K}=\left(K_{i}, \sigma_{i j} \mid K_{i}\right)$ will be a kernel in $C$ if it is an object there. Since $K_{i} \rightarrow K_{i}^{\bullet}$ is a restriction of $M_{i} \rightarrow M_{i}^{\bullet}$, it is injective and so $\mathbf{K}$ is a kernel in $C$.

For cokernel we must use more care. If $p$ is a peak, then $M_{p}^{\bullet}=M_{p}$ and the injective condition is automatic. We want to define $\mathbf{D}=\left(D_{i}, \delta_{i j}\right)$ and $\psi: \mathbf{N} \rightarrow \mathbf{D}$. For $p$ a peak, define $D_{p}=$ Coker $\varphi_{p}=N_{p} / \varphi_{p}\left(M_{p}\right)$, and let $\psi_{p}: N_{p} \rightarrow D_{p}$ be the natural map. We have a commutative diagram

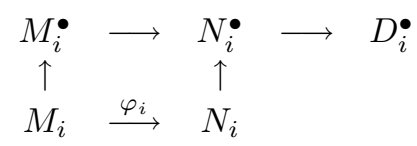

We define $D_{i}=N_{i} / \operatorname{Ker}\left(N_{i} \rightarrow N_{i}^{\bullet} \rightarrow D_{i}^{\bullet}\right)$, and let $\psi_{i}: N_{i} \rightarrow D_{i}$ be the natural map. We now have a natural injection $D_{i} \rightarrow D_{i}^{\bullet}$ such that the new square commutes. The maps $\delta_{i j}$ can be regarded as induced by the projections $D_{i}^{\bullet} \rightarrow D_{j}^{\bullet}$. Thus $\mathbf{D}$ is an object in $C$, and $\psi=$ Coker $\varphi$ since we have taken cokernels $D_{p}$ for peaks $p$, and for $i \in I$ not a peak, $D_{i}$ has been taken universal with respect to $D_{i} \rightarrow D_{i}^{\bullet}$ being injective. Thus we have verified (C2).

If $\left\{\mathbf{M}^{\gamma}: \gamma \in \Gamma\right\}$ is a family in $C$, define $\mathbf{M} \in C$ by $M_{i}=\bigoplus_{\gamma \in \Gamma} M_{i}^{\gamma}$ and $\sigma_{i j}=$ $\bigoplus_{\gamma \in \Gamma} \sigma_{i j}^{\gamma}$. It is easy to see that $\mathbf{M}$ is indeed in $C$, and that with the morphisms $\mathbf{M}^{\gamma} \rightarrow \mathbf{M}$, where $M_{i}^{\gamma} \rightarrow M_{i}$ is the natural injection, $\mathbf{M}$ is a direct sum for the family $\left\{\mathbf{M}^{\gamma}: \gamma \in \Gamma\right\}$. The composition property in (C3) is obvious.

We summarize these important properties of $C$ as a

Proposition 3. The category $P(I, R)$ of peak I-spaces over $R$ forms a pre-module $R$-category with arbitrary direct sums.

Remark. The definition of a pre-module $R$-category is given in the introduction. Simson [53, p. 32] defines cokernels differently and the two notions are the same if the map $\varphi: \mathbf{M} \rightarrow \mathbf{N}$ is "proper" in the sense of Gabriel, which means that a purity condition holds: $M_{i} \varphi_{i}=M_{i}^{\bullet} \varphi_{i}^{\bullet} \cap N_{i}$ for all $i \in I$ (see also [38], Theorem 1.1). In this case the first map is the kernel of the constructed cokernel. However, there are easy morphisms without this property (see [53, p. 32, Example]). Now it will be convenient to show that all kernels in $C$ are semistable.

Lemma 1. All kernels in $C$ are semistable.

Proof. Let

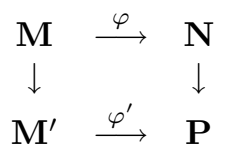


be a pushout diagram in $C$ with $\varphi$ a kernel. The cokernels of $\varphi$ and $\varphi^{\prime}$ can be identified; therefore for every $i \in I$ we obtain a commutative diagram

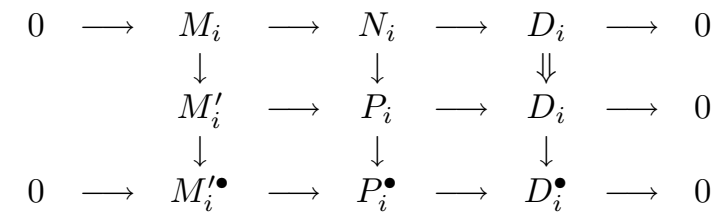

Here $\mathbf{D}$ denotes the cokernel of $\varphi$ and the top row is exact since $\varphi$ is a kernel. To say $\mathbf{P}$ is a pushout is equivalent to

$$
\mathbf{M} \rightarrow \mathbf{M}^{\prime} \oplus \mathbf{N} \rightarrow \mathbf{P} \rightarrow \mathbf{0}
$$

being exact. For peaks $p$, the cokernel is the usual $R$-module cokernel; thus $P_{p}$ is a pushout. This implies that the bottom row of the diagram is exact. The lower vertical arrows are all injections. Since $\mathbf{P}$ is a pushout, $M_{i}^{\prime} \oplus N_{i} \longrightarrow P_{i}$ is surjective; thus $P_{i}$ is generated by the images of $M_{i}^{\prime}$ and $N_{i}$. These facts, plus standard diagram chasing arguments, show that

$$
0 \longrightarrow M_{i}^{\prime} \longrightarrow P_{i} \longrightarrow D_{i} \longrightarrow 0
$$

is exact for every $i \in I$. Thus $\varphi^{\prime}$ is a kernel.

We also have a dual result, which can be shown by dual arguments. It is also mentioned in [38].

Lemma 1*. All cokernels in $C$ are semistable.

Next, we need some $R$-modules which are embedded in torsion-free $R[X]$-modules of rank one. We refer to constructions in [29] for details. Select polynomials $p_{i} \in$ $R[X](i \in \omega)$ as in [29] and let $S$ be the multiplicative set they generate. For $\alpha \subseteq \omega$, define $L_{\alpha}=\left\{\frac{f}{p_{i_{0}} \cdots p_{i_{k}}}\right\} \subseteq S^{-1} R[X]$, where $i_{0}, \ldots, i_{k}$ are distinct elements of $\alpha$, and $f \in R[X]$. Then $L_{\alpha}$ is an $R[X]$-module of $S$-torsion-free rank 1. Define $R$-submodules $U_{\alpha}, V_{\alpha}$ of $L_{\alpha}$ by $U_{\alpha}=\bigoplus_{i \in \alpha}\left(\bigoplus_{0 \leq j<d_{i}} R \cdot \frac{X^{j}}{p_{i}}\right)$ and $V_{\alpha}=U_{\alpha} \oplus R \cdot 1$. Then $U_{\alpha}$ and $V_{\alpha}$ are the rational polynomials in $L_{\alpha}$ of degrees $\leq-1$ and $\leq 0$ respectively. We have a map $X: U_{\alpha} \rightarrow V_{\alpha}, u \rightarrow X \cdot u$.

Lemma 2. Let $N$ be an R-module, $\alpha \cup \beta \subseteq \omega$.

(1) The inclusions $U_{\alpha} \subseteq V_{\alpha} \subseteq L_{\alpha}$ induce injections $N \otimes U_{\alpha} \rightarrow N \otimes V_{\alpha} \rightarrow N \otimes L_{\alpha}$, and $N \otimes L_{\alpha}$ is an $S$-torsion-free $R[X]$-module.

(2) Any R-homomorphism $\varphi: V_{\alpha} \rightarrow N \otimes V_{\beta}$ such that $U_{\alpha} \varphi \subseteq N \otimes U_{\beta}$ and $(X u) \varphi=X(u \varphi)\left(u \in U_{\alpha}\right)$ extends uniquely to an $R[X]$-homomorphism $\widehat{\varphi}: L_{\alpha} \rightarrow$ $N \otimes L_{\beta}$

(3) Let $\varphi$ be as in (2). Then

$$
\varphi=\left\{\begin{array}{l}
n \otimes 1_{\alpha \alpha} \text { for some } n \text { if } \alpha=\beta, \\
0 \quad \text { if } \alpha \backslash \beta \text { is infinite. }
\end{array}\right.
$$

Proof. (1) The $R$-module $U_{\alpha}$ is a summand of $V_{\alpha}$, and $V_{\alpha}$ of $L_{\alpha}$ since

$$
L_{\alpha}=V_{\alpha} \oplus X \cdot R[X] .
$$

Thus the statement on injections follows. If $p_{i}$ is one of the generators of $S$, then by partial fractions $p_{i} \cdot L_{\alpha}$ has an $R$-free complement in $L_{\alpha}$. Thus multiplication 
by $p_{i}: L_{\alpha} \rightarrow L_{\alpha}$ is a split injection; therefore so is $N \otimes L_{\alpha} \rightarrow N \otimes L_{\alpha}$, showing $N \otimes L_{\alpha}$ is $S$-torsion-free.

(2) Since $U_{\alpha} \oplus R[X]=L_{\alpha}$ and $V_{\alpha}=U_{\alpha} \oplus R \cdot 1, \varphi$ extends to a unique $R[X]$ homomorphism $\widehat{\varphi}: L_{\alpha} \rightarrow N \otimes L_{\beta}$ such that $1 \widehat{\varphi}=1 \varphi$.

(3) If $\alpha=\beta$, the type of $L_{\alpha}$ implies $1 \varphi \in N \otimes R[X]$. But $1 \varphi \in N \otimes V_{\beta}$ also; hence $1 \varphi \in N \otimes R$. If $\alpha \backslash \beta$ is infinite, then $s(1 \varphi) \in N \otimes p_{i} R[X]$ for some $s \in S$ and all (infinitely many) $i \in \alpha \backslash \beta$. Thus $1 \varphi=0$ and so $\varphi=0$.

Fix the poset $I$. From now on we assume $I$ is finite. We wish to describe the nature of the construction of a rigid weak $\omega$-family $\left\{\mathbf{X}_{\alpha}: \alpha \subseteq \omega\right\}$ in $C=P(I, R)$ before giving constructions for specific posets. Define the condition

$\left(^{*}\right)$ For each $i \in I$, there is a finite set $\Gamma_{i}$ (disjoint for different $i$ ) with possibly one distinguished member such that if we put $S_{\alpha}^{i}=\bigoplus_{\gamma \in \Gamma_{i}} U_{\alpha}^{\gamma}(\alpha \subseteq \omega)$, where each $U_{\alpha}^{\gamma}=U_{\alpha}$, except for the distinguished $\gamma$, in which case $U_{\alpha}^{\gamma}=V_{\alpha}$, then we have isomorphisms $X_{\alpha}^{i} \rightarrow S_{\alpha}^{i}$ such that for $\alpha \subseteq \beta$ we have a commutative diagram

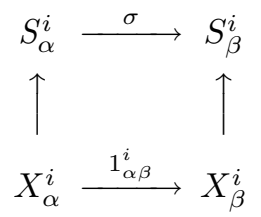

where $\sigma: S_{\alpha}^{i} \rightarrow S_{\beta}^{i}$ is induced by $U_{\alpha} \subseteq U_{\beta}, V_{\alpha} \subseteq V_{\beta}$.

Let $N$ be an $R$-module and $\alpha \cup \beta \subseteq \omega$. Define $H_{\alpha \beta}^{N}=\left\{f \in \operatorname{Hom}_{R}\left(V_{\alpha}, N \otimes V_{\beta}\right): f\right.$ satisfies Lemma 2(2)\}; thus $U_{\alpha} f \subseteq N \otimes U_{\beta}$ and $(X u) f=X(u f)$. For all $i \in I$, the map $f$ induces $S_{\alpha}^{i} \rightarrow N \otimes S_{\beta}^{i}$, which the isomorphisms of (*) carry to $X_{\alpha}^{i} \rightarrow N \otimes X_{\beta}^{i}$.

We define condition

(**) The maps $X_{\alpha}^{i} \rightarrow N \otimes X_{\beta}^{i}$ induced by $f$ in turn induce a morphism $\varphi_{f}: \mathbf{X}_{\alpha} \rightarrow N \otimes \mathbf{X}_{\beta}$. The map $f \rightarrow \varphi_{f}: H_{\alpha \beta}^{N} \rightarrow \operatorname{Mor}\left(\mathbf{X}_{\alpha}, N \otimes \mathbf{X}_{\beta}\right)$ is bijective.

Lemma 3. If $\left\{\mathbf{X}_{\alpha}: \alpha \subseteq \omega\right\}$ satisfies $\left(^{*}\right)$ and (**), then it is a rigid weak $\omega$-family in $C$.

Proof. Taking $N=R$ and $f$ to be the inclusion $V_{\alpha} \subseteq V_{\beta}$, we obtain the morphisms $1_{\alpha \beta}: \mathbf{X}_{\alpha} \longrightarrow \mathbf{X}_{\beta}$ from (**) making $\left\{\mathbf{X}_{\alpha}: \alpha \subseteq \omega\right\}$ into an $\omega$-family. Since for arbitrary $\alpha \cup \beta \subseteq \omega$, we have $U_{\alpha}+U_{\beta}=U_{\alpha \cup \beta}$ and $U_{\alpha} \cap U_{\beta}=U_{\alpha \cap \beta}$, then we have an exact sequence $0 \rightarrow U_{\alpha \cap \beta} \rightarrow U_{\alpha} \oplus U_{\beta} \rightarrow U_{\alpha \cup \beta} \rightarrow 0$ and a similar statement for $V_{\alpha}$. By $\left(^{*}\right)$, this induces $0 \rightarrow X_{\alpha \cap \beta}^{i} \rightarrow X_{\alpha}^{i} \oplus X_{\beta}^{i} \rightarrow X_{\alpha \cup \beta}^{i} \rightarrow 0$ for all $i \in I$, which by (**) induces $0 \rightarrow \mathbf{X}_{\alpha \cap \beta} \rightarrow \mathbf{X}_{\alpha} \oplus \mathbf{X}_{\beta} \rightarrow \mathbf{X}_{\alpha \cup \beta} \rightarrow 0$, where the maps are $1_{\alpha \cap \beta, \alpha} \oplus 1_{\alpha \cap \beta, \beta}$ and $1_{\alpha, \alpha \cup \beta}+1_{\beta, \alpha \cup \beta}$. Thus we have a weak $\omega$-family.

We must verify (W1)-(W4) for every $R$-module $N$. We have already shown in Lemma 1 that all kernels are semistable in this category. Since, for $\alpha \subseteq \beta, U_{\alpha} \rightarrow U_{\beta}$ is a split injection in the category of $R$-modules, the same holds for $X_{\alpha}^{i} \rightarrow X_{\beta}^{i}$; thus $N \otimes_{-}$is exact on the sequences above. This shows that $N \otimes \mathbf{X}_{\alpha \cap \beta} \rightarrow N \otimes\left(\mathbf{X}_{\alpha} \oplus \mathbf{X}_{\beta}\right)$ is a kernel, and (W1) follows. Let $\varphi: \mathbf{X}_{\alpha} \rightarrow \bigoplus_{\lambda}\left(N \otimes \mathbf{X}_{\beta}\right)$ be a morphism. Since $\mathbf{X}_{\alpha}$ is determined by finitely many copies of $U_{\alpha}$ or $V_{\alpha}$, for all but finitely many indices in $\lambda$, all these copies map to torsion since, by $\left(^{* *}\right)$ and Lemma 2 , they come from $R[X]$-homomorphisms on $L_{\alpha}$, which is rank 1 . But $N \otimes L_{\beta}$ is a torsion-free $R[X]$-module by Lemma 2 ; thus $\varphi$ factors through a finite direct sum in $\oplus_{\lambda}$. (W2) is therefore shown. (W3) follows for a similar reason, since $1_{\emptyset \alpha} \circ \varphi=0$ implies that 
$\varphi$ maps $X_{\alpha}$ into torsion, thus to 0 . Finally (W4) comes immediately from (**) and Lemma 2(3).

In the remainder of this section, we verify the existence of rigid weak $\omega$-families for the posets from the list in the Appendix. This will give us

Theorem 2. Let $R$ be a commutative ring with $0 \neq 1$ and $I$ be a finite poset containing as a full peak subposet, one of the 114 posets in Simson's list from the Appendix $\S 7$. Let $\lambda$ be an infinite cardinal and $A$ an $R$-algebra that can be generated by $\leq \lambda$ elements. Then there exists a $\lambda$-family of functors from $A$-Mod to $P(I, R)$, the category of peak I-spaces over the commutative ring $R$, which is rigid for every $A$-module. That is, for any $A$-modules $M$ and $N$, and $\alpha \cup \beta \subseteq \lambda$,

$$
\operatorname{Mor}\left(\mathbf{M}_{\alpha}, \mathbf{N}_{\beta}\right)=\left\{\begin{array}{l}
\operatorname{Hom}_{A}(M, N) \text { if } \alpha \subseteq \beta, \\
0 \text { if } \alpha \nsubseteq \beta
\end{array}\right.
$$

It will be useful to know that finding appropriate families in $C=P(I, R)$ may yield appropriate families in $C^{\prime}=P\left(R, I^{\prime}\right)$ for a related poset $I^{\prime}$.

Let us say that $A=\left\{i_{1}, \ldots, i_{k}\right\} \subseteq I(k \geq 2)$ is an arm at $m \in I$ if $i_{1}<i_{2}<\ldots$ $<i_{k}=m$, and if any $j \in I \backslash A$ is related to an element $i_{l}$ of $A$, then either $j>i_{k}$ or else $l=k$.

We define a new ordering on $I$, call $I^{\prime}$ the new poset, by deleting all relations involving $i_{1}, \ldots, i_{k-1}$ and any element outside $\left\{i_{1}, \ldots, i_{k-1}\right\}$, and adding new relations $i_{k-1}>j$ for all $j \in I$ with $j \leq i_{k}$. We refer to this alteration of $I$ as "bending an arm".

Before giving a condition on an arm, we note that if $i<j$ such that the peaks above $i$ are the same as those above $j$, i.e. $I_{i}=I_{j}$, and if $\mathbf{M}$ is a peak $I$-space, then the map $M_{i} \rightarrow M_{j}$ is injective. In particular, this applies if $i$ and $j$ lie on an arm of $I$.

For simplicity of notation, suppose that $1<2<\ldots<k$ is an arm at $k$. For this arm, we define the condition

$\left.{ }^{* * *}\right)$ For $1 \leq i \leq k-1$, there is a subset $\Gamma_{i+1}^{\prime} \subseteq \Gamma_{i+1}$ such that for all $\alpha \subseteq \omega$, the image of $X_{\alpha}^{i} \rightarrow X_{\alpha}^{i+1} \rightarrow S_{\alpha}^{i+1}$ has a complement

$$
\bigoplus_{\gamma \in \Gamma_{i+1}^{\prime}} U_{\alpha}^{\gamma} \text { in } S_{\alpha}^{i+1}=\bigoplus_{\gamma \in \Gamma_{i+1}} U_{\alpha}^{\gamma}
$$

Lemma 4. Let $I$ be a finite poset and $I^{\prime}$ a poset obtained from $I$ by one of the following:

(a) There is an $m \in I$ which is not a peak, and an arm at $m$ is bent; or

(b) There is peak $m \in I$ and two distinct arms at $m$ are bent.

Assume $\left\{\mathbf{X}_{\alpha}: \alpha \subseteq \omega\right\}$ is a family in $P(I, R)$ satisfying $\left(^{*}\right)$ and $\left({ }^{* *}\right)$, and such that the arms in $(a)$ or (b) satisfy (***), and in the case of $(b)$, if $i_{1}$ and $j_{1}$ are the smallest vertices on the two arms, then $X_{\alpha}^{i_{1}} \cap X_{\alpha}^{j_{1}}=0$ (when both embedded in $\left.X_{\alpha}^{m}\right)$.

Then there is a family in $P\left(I^{\prime}, R\right)$ satisfying $(*)$ and $\left({ }^{*}\right)$, and satisfying $\left({ }^{* *}\right)$ for any arm of $I^{\prime}$ such that the corresponding arm of I satisfies $(* * *)$.

Proof. Suppose first that we are in (a), and the arm at $k$ is $1<2<\ldots<k$. Define $\mathbf{Y}_{\alpha} \in P\left(I^{\prime}, R\right)$ by $Y_{\alpha}^{i}=X_{\alpha}^{i}$ for $i \notin\{1, \ldots, k-1\}$ and let maps $Y_{\alpha}^{i} \rightarrow Y_{\alpha}^{j}$ be $X_{\alpha}^{i} \rightarrow X_{\alpha}^{j}$. For $i \in\{1, \ldots, k-1\}$, regarding $X_{\alpha}^{1}$ as embedded in $X_{\alpha}^{i+1}$, define $Y_{\alpha}^{i}=X_{\alpha}^{i+1} / X_{\alpha}^{1}$. By $(* * *)$ there is a natural isomorphism $Y_{\alpha}^{i} \rightarrow \bigoplus_{\gamma \in \Gamma_{2}^{\prime} \cup \ldots \cup \Gamma_{i+1}^{\prime}} U_{\alpha}^{\gamma}=S_{\alpha}^{\prime i}$. 
If $i, j \in\{1, \ldots, k-1\}, i<j$, then $Y_{\alpha}^{i} \rightarrow Y_{\alpha}^{j}$ is the natural map, forming a commutative square with the natural mapping $S_{\alpha}^{\prime}{ }^{i} \rightarrow S_{\alpha}^{\prime}{ }^{j}$. The map $Y_{\alpha}^{k}=X_{\alpha}^{k} \rightarrow$ $X_{\alpha}^{k} / X_{\alpha}^{1}=Y_{\alpha}^{k-1}$ is the natural map, which commutes with the projection $S_{\alpha}^{k} \rightarrow$ $S_{\alpha}^{\prime{ }^{k-1}}$. For completeness, if $j \in I^{\prime} \backslash\{1, \ldots, k\}$ and $j<k$, then we must specify $Y_{\alpha}^{j} \rightarrow Y_{\alpha}^{k-1}$. Maps $Y_{\alpha}^{j} \rightarrow Y_{\alpha}^{k-1}$ for $j \in I^{\prime} \backslash\{1, \ldots, k\}$ and $j<k$, are obtained by mapping through $Y_{\alpha}^{k}$. Since the index sets in (***) are independent of $\alpha$, we see that $\left(^{*}\right)$ is satisfied by $\left\{\mathbf{Y}_{\alpha}: \alpha \subseteq \omega\right\}$. To check (***) for $\left\{\mathbf{Y}_{\alpha}: \alpha \subseteq \omega\right\}$, we only need consider the new arm created by bending. A complement for $Y_{\alpha}^{i} \rightarrow S_{\alpha}^{\prime i+1}(i \leq k-2)$ is clearly given by $\bigoplus_{\gamma \in \Gamma_{i+2}^{\prime}} U_{\alpha}^{\gamma}$. Therefore $\left({ }^{* *}\right)$ is maintained. To observe that $\mathbf{Y}_{\alpha}$ really lies in $P\left(I^{\prime}, R\right)$, we have to check the injectivity of $Y_{\alpha}^{i} \rightarrow Y_{\alpha}^{i \bullet}$. For $i \in\{1, \ldots, k-1\}$, this is already obvious since $Y_{\alpha}^{i \bullet}=Y_{\alpha}^{k-1}$. For other $i$, the peaks of $I^{\prime}$ dominating $i$ are those of $I$ dominating $i$, with possibly the addition of $k-1$. Thus $Y_{\alpha}^{i} \rightarrow Y_{\alpha}^{i \bullet}$ is injective.

To check (**), let $N$ be an $R$-module and $\alpha \cup \beta \subseteq \omega$. If $f \in H_{\alpha \beta}^{N}$, then $f$ induces a morphism $\varphi_{f}: \mathbf{Y}_{\alpha} \rightarrow N \otimes \mathbf{Y}_{\beta}$ since $\left(^{*}\right)$ is satisfied. Let $\varphi: \mathbf{Y}_{\alpha} \rightarrow N \otimes \mathbf{Y}_{\beta}$ be a morphism. We must show that $\varphi$ is obtained in this fashion. If $i \in\{1, \ldots, k-2\}$, then the fact that the maps $Y_{\alpha}^{i}=X_{\alpha}^{i+1} / X_{\alpha}^{1} \rightarrow X_{\alpha}^{k} / X_{\alpha}^{1}=Y_{\alpha}^{k-1}$ and $Y_{\alpha}^{k}=X_{\alpha}^{k} \rightarrow$ $X_{\alpha}^{k} / X_{\alpha}^{1}=Y_{\alpha}^{k-1}$ are the natural maps, and similarly for $N \otimes \mathbf{Y}_{\beta}$, shows that the morphism $\varphi$ must satisfy $X_{\alpha}^{i} \varphi \subseteq N \otimes X_{\beta}^{i}$ for $i=1$ on $X_{\alpha}^{k}=Y_{\alpha}^{k}$, and thus holds for $i \in\{1, \ldots, k-1\}$. Thus $\varphi$ may be regarded as a morphism $\mathbf{X}_{\alpha} \rightarrow N \otimes \mathbf{X}_{\beta}$, which must be of form $\varphi_{f}$ on $\mathbf{X}_{\alpha}$ by $\left.{ }^{* *}\right)$. By the way $S_{\alpha}^{\prime i}$ is given, the map $\varphi^{i}: Y_{\alpha}^{i} \rightarrow N \otimes Y_{\beta}^{i}$ is induced by $f$ on $S_{\alpha}^{\prime i}$; thus we have shown (**) holds for $\left\{\mathbf{Y}_{\alpha}: \alpha \subseteq \omega.\right\}$

In the case of (b), the argument for two arms at $m$ goes through similarly, except that one must check the injectivity condition $Y_{\alpha}^{i} \rightarrow Y_{\alpha}^{i \bullet}$ differently. The question is for $i \in I^{\prime}$ not in either arm. If $i_{k-1}$ and $j_{\ell-1}$ are the two arm vertices just below $m$, then the peak $m \in I$ is replaced by peaks $i_{k-1}$ and $j_{\ell-1}$ in $I^{\prime}$. The map $Y_{\alpha}^{m} \rightarrow Y_{\alpha}^{i_{k-1}} \oplus Y_{\alpha}^{i_{l-1}}$ is $X_{\alpha}^{m} \rightarrow X_{\alpha}^{m} / X_{\alpha}^{i_{k}} \oplus X_{\alpha}^{m} / X_{\alpha}^{j}$ which is injective by the assumption in (b). Thus $Y_{\alpha}^{i} \rightarrow Y_{\alpha}^{i \bullet}$ will be injective since $X_{\alpha}^{i} \rightarrow X_{\alpha}^{i \bullet}$ is.

To describe families $\left\{\mathbf{X}_{\alpha}: \alpha \subseteq \omega\right\}$ in $P(I, R)$ satisfying $\left({ }^{*}\right)$ and $(* *)$ we need terminology and notation for simplification. The subscript $\alpha(\alpha \subseteq \omega)$ will be dropped except when necessary. The components of $\mathbf{X}$ will be isomorphic to either $U_{\alpha}$ or $V_{\alpha}$, and will be denoted by symbols such as $1,2, \ldots, A, B, \ldots$. Only one component will be isomorphic to $V_{\alpha}$; if it is $\mathrm{A}$, we write $\mathbf{A}$ at one point to identify it. $X^{\bullet}$ will be the direct sum of the components of $\mathbf{X}$. We further define certain $R$-submodules of $X$ ' we call basic submodules. If $A_{1}, \ldots, A_{k}$ are distinct components of $X$, we let $A_{1} \ldots A_{k},\left(k \geq 2\right.$ and $\left.A_{1}=\mathbf{A}_{1}\right), A_{1}+\ldots+A_{k}$, and $X A_{1}+A_{2}+\ldots+A_{k}(k \geq 2)$ denote the submodules of $X \bullet$ given by:

$$
\begin{gathered}
A_{1} \ldots A_{k}=A_{1} \oplus \ldots \oplus A_{k}, \\
A_{1}+\ldots+A_{k}=\left\{u_{1}+\ldots+u_{k}: u_{i}=u \in U\right\}, \\
X A_{1}+\ldots+A_{k}=\left\{X u_{1}+u_{2}+\ldots+u_{k}: u_{i}=u \in U\right\} .
\end{gathered}
$$

To put a $P(I, R)$-structure on $X^{\bullet}$, we must define $R$-modules $X^{i}$ for all $i \in I$. Each $X^{i}$ will be a direct sum of basic submodules. The mappings $X^{i} \rightarrow X^{j}$ will come from natural inclusions and projections.

We call a submodule $T$ of $X \bullet$ invariant if it is expressible by finite sums and intersections of the $X^{i}(i \in I)$. If $T \subseteq X^{\bullet}$ is invariant, then $N \otimes T$ is invariant in 
$N \otimes X^{\bullet}$. If $\varphi: \mathbf{X}_{\alpha} \rightarrow N \otimes \mathbf{X}_{\beta}$ is a morphism in $P(I, R)$, then $T_{\alpha} \varphi \subseteq N \otimes T_{\beta}$ if $T$ is invariant in $X^{\bullet}$.

If $\alpha \subseteq \beta$, the inclusions $U_{\alpha} \subseteq U_{\beta}$ and $V_{\alpha} \subseteq V_{\beta}$ induce an inclusion $X_{\alpha}^{\bullet} \rightarrow X_{\beta}^{\bullet}$. From this and the form of the basic submodules, we see that $\left(^{*}\right)$ is immediate. To show that $\left({ }^{* *}\right)$ is satisfied, by standard arguments it suffices to show that

(a) all components and basic submodules in the presentation of $X^{\bullet}$ are invariant,

(b) all components are "linked", i.e. $A+B$ is invariant for all components $A$ and $B$, and

(c) the basic submodule $X A_{1}+\ldots+A_{k}$ occurs.

To see that this suffices, we note that $A_{1}=\mathbf{A}_{1}$, and that $A_{1}, A_{2}, A_{1}+A_{2}$ and $X A_{1}+A_{2}$ are invariant. This determines a map $f \in \operatorname{Hom}_{R}\left(V_{\alpha}, N \otimes V_{\beta}\right)$. Since all components are linked, $\varphi=\varphi_{f}$, and $\left({ }^{*}\right)$ will hold. We now consider the posets $\mathcal{P}_{n}$ enumerated by Simson [54] and given in the Appendix of this paper. For each $\mathcal{P}_{n}$, we list on the first line, contrary to the appendix where the orderings are drawn upside-down, the $X^{p}$ for $p$ a peak and arrows will indicate the ordering. If it is necessary to check condition (***) for an arm, it will follow from the simple form of the basic submodules. If we want to sum two $X^{i}$, we will use $\oplus$ to avoid confusion in the basic submodule use of + .

Now we begin distinguishing 19 "equivalence classes" under bending arms of Simson's 114 cases. There is no general rule for obtaining the peak $I$-spaces presented below. To help the reader, in Case $2 \mathrm{c}$ we give an explicit description of the object defined there.

Case 1. $\mathcal{P}_{1, n}(n \geq 0)$.

$$
\begin{aligned}
& \uparrow^{\mathbf{1}} \nearrow^{2} \nwarrow \nearrow^{\cdots}{ }^{\cdots} \nearrow^{n+2} \nwarrow^{\mathbf{1}} \\
& 1+2,2+3, \ldots,(n+1)+(n+2), X 1+(n+2) \text {. }
\end{aligned}
$$

It is clear (a)-(c) hold in this case.

Case 2. $\mathcal{P}_{3, n}^{\prime}(n \geq 1)$.

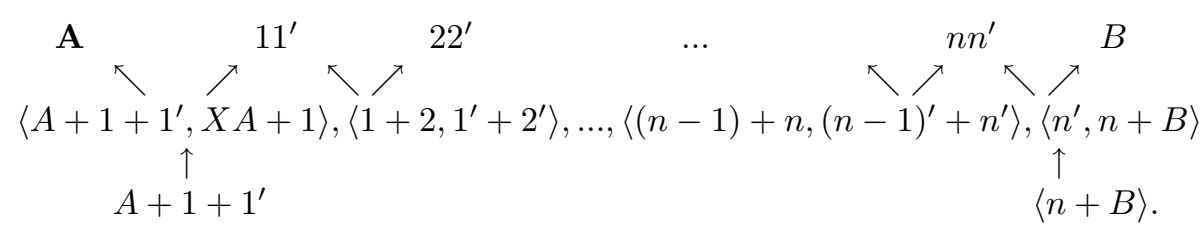

To verify (a), we note $n$ is invariant and $n^{\prime}=n n^{\prime} \cap\left\langle n^{\prime}, n+B\right\rangle$; thus $n^{\prime}$ is invariant. Moreover $(n-1)=\left(\left\langle(n-1)+n,(n-1)^{\prime}+n^{\prime}\right\rangle \oplus n\right) \cap(n-1)(n-1)^{\prime}$. Inductively we may show all the components are invariant. Now (b) and (c) follow easily.

Case 2a. The missing case $\mathcal{P}_{3,0}^{\prime}$ is Case 2c.

By bending an arm of $\mathcal{P}_{3, n}^{\prime}(n \geq 1)$, and applying Lemma 4, we take care of

Case 2b. $\mathcal{P}_{3, n}(n \geq 0)$.

We take care of $\mathcal{P}_{3,0}^{\prime}$ separately. 
Case 2c. $\mathcal{P}_{3,0}^{\prime}$.

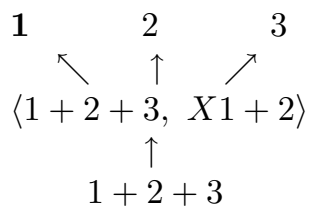

(a)-(c) follow easily. To describe the object constructed, at the peaks we have $X^{\bullet}=V \oplus U \oplus U$. At the middle vertex we have the (inner direct) sum of the basic submodules $\{(u, u, u): u \in U\}$ and $\{(X u, u, 0): u \in U\}$. At the bottom vertex is the basic submodule $\{(u, u, u): u \in U\}$. The maps are inclusion and projections.

By now bending an arm of $\mathcal{P}_{3, n}$ and applying Lemma 4 , we take care of

Case 2d. $\mathcal{P}_{2, n}(n \geq 0)$.

Case 3. $\mathcal{P}_{2, n+1}^{\prime}(n \geq 0)$.

Case 3a. Here we deal with the poset $\mathcal{P}_{4}$ (see [29]), because it can be viewed as $\mathcal{P}_{2,0}^{\prime}$ and it can be handled by a variation of $\mathcal{P}_{1,2}$, namely

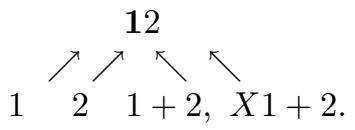

Case 3 b. For $n \geq 1$, we use

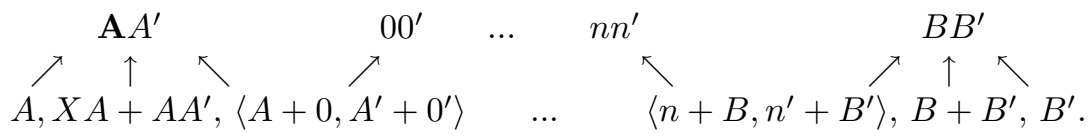

The problem is to show all the components are invariant.

$0=\left(\left\langle A+0, A^{\prime}+0^{\prime}\right\rangle \oplus A\right) \cap 00^{\prime}$; thus 0 is invariant. Similarly, we show $1, \ldots, n, B$ are invariant. Starting with $B^{\prime}$ and working the other direction, we show $n^{\prime}, \ldots, 0^{\prime}, A^{\prime}$ are invariant.

Case 4. $\mathcal{P}_{3, n}^{\prime \prime}(n \geq 0)$.

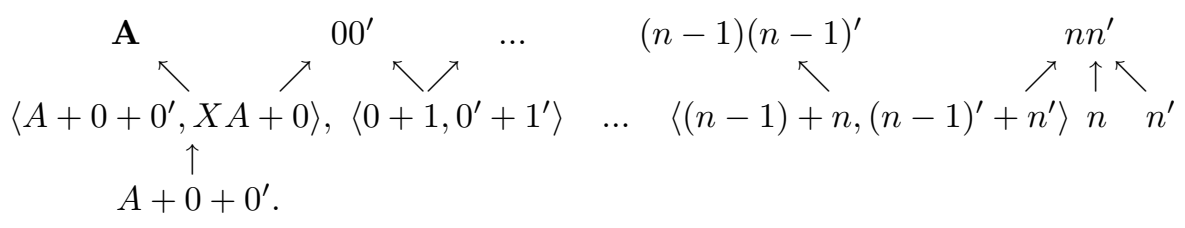

By methods similar to the above and starting with $n$ and $n^{\prime}$, we show all components are invariant. $0, \ldots, n$ are linked, as are $0^{\prime}, \ldots, n^{\prime}$. But $A$ is linked to both 0 and $0^{\prime}$. Applying Lemma 4 to $\mathcal{P}_{3, n}^{\prime \prime}$, we take care of

Case 4a. $\mathcal{P}_{2, n}^{\prime \prime}$.

Case 5. $\mathcal{P}_{5}$.

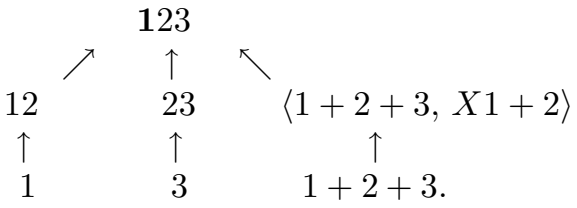


This is easy since $12 \cap 23=2$.

By applications of Lemma 4 to $\mathcal{P}_{5}$, we take care of

Case 5 a. $\mathcal{P}_{n}$ for $n=9,10,32-35$.

Case 6. $\mathcal{P}_{6}$.

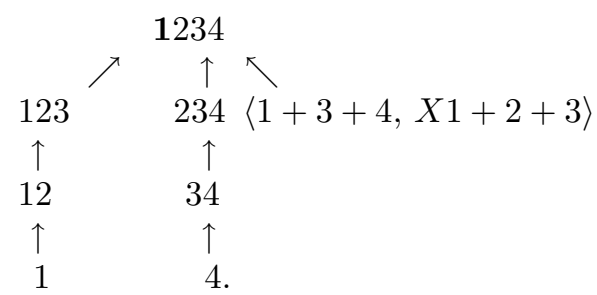

One sees that $2=12 \cap 234,3=123 \cap 34$. By Lemma 4 , we also take care of

Case 6a. $\mathcal{P}_{n}$ for $n=12-15,37-46,50-52$.

Case 7. $\mathcal{P}_{7}$.

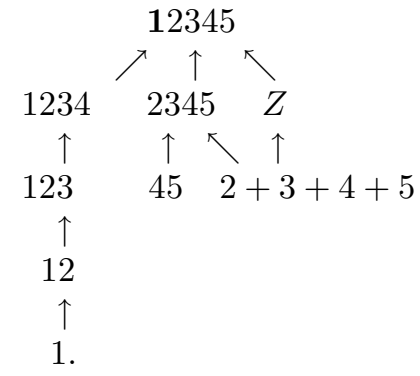

Let $Z=\langle 2+3+4+5,1+5, X 1+3\rangle$. Then $1+5=Z \cap(1 \oplus 45), X 1+3=Z \cap(123)$, $2=12 \cap 2345,4=1234 \cap 45,3=((X 1+3) \oplus 1) \cap 2345$, and $5=((1+5) \oplus 1) \cap 45$.

By Lemma 4, we also take care of

Case 7 a. $\mathcal{P}_{n}$ for $n=17,18,22,29,58,80-83$.

Case $7 \mathrm{~b} . \mathcal{P}_{21}$.

This case can be obtained from $\mathcal{P}_{7}$ as follows. In $\mathcal{P}_{7}$ delete the arrow coming from the inclusion $2+3+4+5 \rightarrow 2345$. The family for $\mathcal{P}_{7}$ is also a family for this new poset satisfying (a)-(c). Now bend the two arms at 12345 whose lowest vertices are 1 and 45 . The projection of 12345 to the new peak $12345 / 45$ restricts to the natural map of $2+3+4+5$ into $2345 / 45$, thus realizing $\mathcal{P}_{21}$.

By Lemma 4, we also take care of

Case $7 \mathrm{c} . \mathcal{P}_{56}$ and $\mathcal{P}_{57}$. 
Case 8. $\mathcal{P}_{8}$.

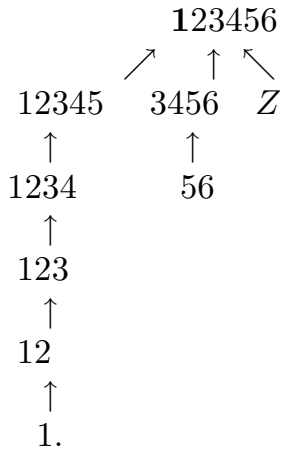

Let $Z=\langle 1+4+5,2+3+5, X 1+3+6\rangle$. Note 3 and 5 are invariant and $34=1234 \cap 3456$. Then $1+4+5=Z \cap(1 \oplus 34 \oplus 5), 2+3+5=Z \cap(123 \oplus 5)$, $X 1+3+6=Z \cap(1 \oplus 3 \oplus 56), 2=((2+3+5) \oplus 3 \oplus 5) \cap 12,4=((1+4+5) \oplus 1 \oplus 5) \cap 34$ and $6=((X 1+3+6) \oplus 1 \oplus 3) \cap 56$.

By Lemma 4, we also take care of

Case 8a. $\mathcal{P}_{n}$ for $n=16,24-28,30,31,61-79,84-99$.

Case 9. $\mathcal{P}_{11}$.

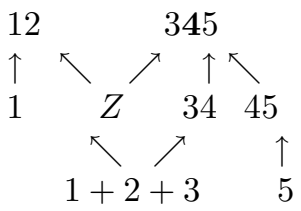

Let $Z=\langle 1+2+3,3+4+5,2+X 4\rangle$.

We observe that $4=34 \cap 45,3+4+5=Z \cap(34 \oplus 5), 2+X 4=Z \cap(12 \oplus 4)$, $3=((1+2+3) \oplus 12) \cap 34$, and $2=((2+X 4) \oplus 4 \cap 12$.

By Lemma 4, we also take care of

Case 9a. $\mathcal{P}_{36}$.

Case 10. $\mathcal{P}_{19}$.

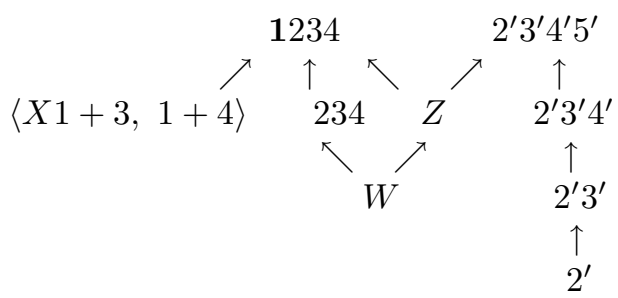

Let $W=\left\langle 2+2^{\prime}+3+3^{\prime}+5^{\prime}, 2+2^{\prime}+4+4^{\prime}\right\rangle$ and $Z=\left\langle 1,2+2^{\prime}, 3+3^{\prime}, 4+4^{\prime}, 5^{\prime}\right\rangle$.

It will suffice to show invariance of all the components. $1=Z \cap 1234$ and $5^{\prime}=Z \cap 2^{\prime} 3^{\prime} 4^{\prime} 5^{\prime}$.

$2=\left(Z \oplus 2^{\prime}\right) \cap 234 . \quad 3+3^{\prime}=\left(\langle X 1+3,1+4\rangle \oplus 1 \oplus 2^{\prime} 3^{\prime}\right) \cap\left(W \oplus\left(2+2^{\prime}\right) \oplus\right.$ $\left.5^{\prime}\right)$. Therefore 3 and $3^{\prime}$ are invariant by using the two peaks. We have $4+4^{\prime}=$ $\left(W \oplus\left(2+2^{\prime}\right)\right) \cap\left(\langle X 1+3,1+4\rangle \oplus 1 \oplus 2^{\prime} 3^{\prime} 4^{\prime}\right)$; thus 4 and $4^{\prime}$ are invariant, and we are done. 
By Lemma 4, we also take care of

Case 10a. $\mathcal{P}_{53}$ and $\mathcal{P}_{54}$.

Case 11. $\mathcal{P}_{20}$.

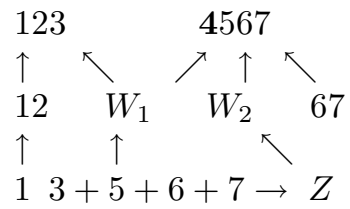

Let $Z=\langle 4+7,5+6+7\rangle, \quad W_{1}=\langle 4,1+5,2+6,3+5+6+7\rangle$ and $W_{2}=$ $\langle X 4+6,4+7,5+6+7\rangle$.

Now we have $2+6=W_{1} \cap(12 \oplus 67)$; thus 2 and 6 are invariant by using the peaks. Similarly 3 is invariant since $3+5+6+7$ is. $4=W_{1} \cap 4567$. Note that $567=(((3+5+6+7) \oplus 123) \cap 4567) \oplus 67$; thus $5=\left(W_{1} \oplus 1\right) \cap 567$. Finally, $7=(\langle 4+7,5+6+7\rangle \oplus 4) \cap 67$.

By Lemma 4, we also take care of

Case 11a. $\mathcal{P}_{55}$.

Case 12. $\mathcal{P}_{23}$.

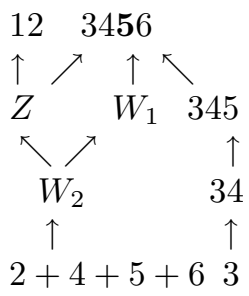

Let $W_{1}=\langle 4+5+6,3+6,3+X 5\rangle, W_{2}=\langle 2+4+5+6,1+3+6\rangle$, and $Z=\langle 1+3,2+4,5,6\rangle$.

We see that $1=(Z \oplus 3) \cap 12,2$ comes from $2+4+5+6$ and the peaks, $4=(Z \oplus 2) \cap 34$, and $5=Z \cap 345$. Finally, $6=\left(W_{2} \oplus(Z \cap 13)\right) \cap 3456$.

Lemma 4 also takes care of

Case $12 \mathrm{a}$. $\mathcal{P}_{n}$ for $n=59,60,103-105$.

Case 13. $\mathcal{P}_{47}$.

$$
\begin{array}{cccc}
1 & & 23 & 4 \\
\uparrow & \nearrow & \uparrow & \uparrow \\
\langle 1+3,2\rangle & X 2+3 & \langle 2+4,3\rangle \\
& & & \nearrow \\
1+2+3+4 &
\end{array}
$$

We see that $2=\langle 1+3,2\rangle \cap 23$ and $3=\langle 2+4,3\rangle \cap 23$ and the rest follows easily. Case 14. $\mathcal{P}_{48}$.

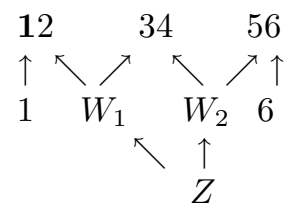


Let $Z=\langle 2+3+6,1+4+5\rangle, W_{1}=\langle 1+4,2+3, X 1+3\rangle$ and $W_{2}=\langle 3+6$, $4+5,3+5\rangle$.

Note that $2+3+6=Z \cap 12346$ and $1+4+5=Z \cap 13456$. Thus $2=$ $((2+3+6) \oplus 3456) \cap 12$, and similar arguments show that 3,4 , and 5 are invariant.

Case $15 . \mathcal{P}_{49}$.

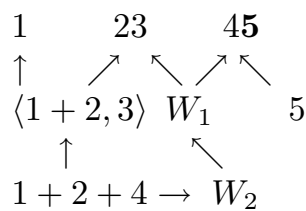

Let $W_{1}=\langle 2+4,3+4+5,4+X 5\rangle$ and $W_{2}=\langle 2+4,3+4+5\rangle$.

Then $2=((1+2+4) \oplus 145) \cap 23,3=\langle 1+2,3\rangle \cap 23$, and $4=((1+2+4) \oplus 12) \cap 45$.

Case 16. $\mathcal{P}_{100}$.

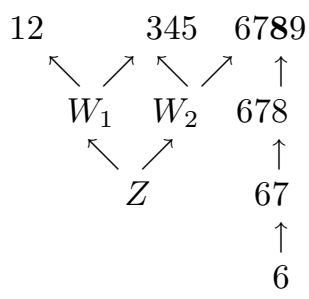

Let $W_{1}=\langle 134,2+5\rangle, W_{2}=\langle 589,3+6,4+7\rangle$, and $Z=\langle 2+5+9,3+4+6+$ $7+8+9,1+4+7+X 8\rangle$.

Clearly, $1=W_{1} \cap 12$ and $5=W_{2} \cap 345$. We note that $34=W_{1} \cap 345$ and $89=W_{2} \cap 6789$. Thus $8=678 \cap 89,3=\left(W_{2} \oplus 6\right) \cap 34,2=(Z \oplus 589) \cap 12$, $7=(Z \oplus 1348) \cap 67,4=\left(W_{2} \oplus 7\right) \cap 34$, and $9=(Z \oplus 25) \cap 89$.

Lemma 4 also takes care of

Case 16a. $\mathcal{P}_{101}$ and $\mathcal{P}_{102}$

Case 17. $\mathcal{P}_{106}$.

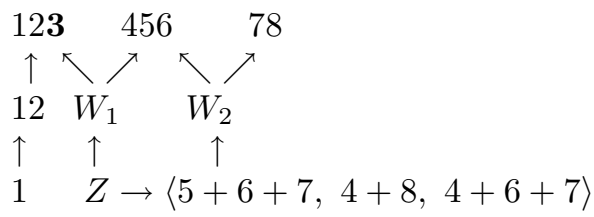

Let $W_{1}=\langle 36,2+4,1+5\rangle, W_{2}=\langle 458,6+7\rangle$ and $Z=\langle 1+3+5+6+7$, $2+X 3+4+8\rangle$.

We see easily that $3=W_{1} \cap 123,6=W_{1} \cap 456 ; 8=W_{2} \cap 78,7=(Z \oplus 13456) \cap 78$, and $4=(Z \oplus 1238) \cap 456$. Then $2=\left(W_{1} \oplus 4\right) \cap 12$.

Finally, note that $56=\left(W_{1} \oplus 1\right) \cap 456$; thus $5=W_{2} \cap 56$.

Lemma 4 also takes care of

Case 17a. $\mathcal{P}_{107}$. 
Case 18. $\mathcal{P}_{108}$

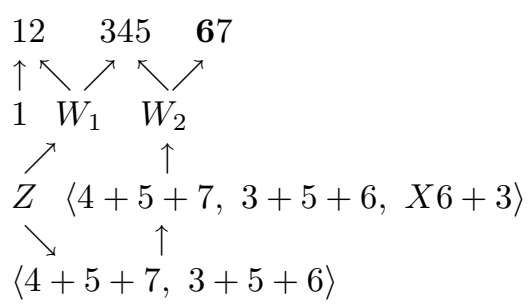

Let $W_{1}=\langle 4,1+3,2+5\rangle, W_{2}=\langle 356,4+7\rangle$ and $Z=2+4+5+7$.

Then $2=(Z \oplus 34567) \cap 12,4=W_{1} \cap 345,6=W_{2} \cap 67$ and $7=(Z \oplus 12345) \cap 67$.

Noting $35=W_{2} \cap 345$, we obtain $3=\left(W_{1} \oplus 1\right) \cap 35$ and $5=(Z \oplus 247) \cap 35$.

Case 19. $\mathcal{P}_{110}$.

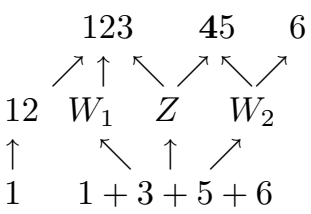

Let $W_{1}=\langle 2,1+3\rangle, Z=\langle 1+3+5,3+4,2+X 4+5\rangle$ and $W_{2}=\langle 4,5+6\rangle$.

Then

$$
\begin{gathered}
2=W_{1} \cap 12, \quad 4=W_{2} \cap 45, \quad 5=((1+3+5+6) \oplus 1236) \cap 45, \\
13=((1+3+5+6) \oplus 156) \cap 123
\end{gathered}
$$

and

$$
3=(Z \oplus 4) \cap 13
$$

Lemma 4 also takes care of

Case 19a. $\mathcal{P}_{109}$

7. Appendix: Simson's minimal posets $\mathcal{P}_{1}, \ldots, \mathcal{P}_{110}$ OF INFINITE PRINJECTIVE TYPE

The system of numbers at a poset refers to its coordinate vector (see [53]). The numbers indicate "dimension," where $U$ and $V$ have dimension one. Those at the peaks are the dimensions there, while the dimensions at other vertices are the sums of the numbers lying at or below the vertex. 
Part 1. The infinite series $\mathcal{P}_{1, n}, \mathcal{P}_{2, n+1}, \mathcal{P}_{2, n+1}^{\prime}, \mathcal{P}_{2, n}^{\prime \prime}, \mathcal{P}_{3, n}, \mathcal{P}_{3, n}^{\prime}, \mathcal{P}_{3, n}^{\prime \prime}$,

$$
n \geq 0
$$
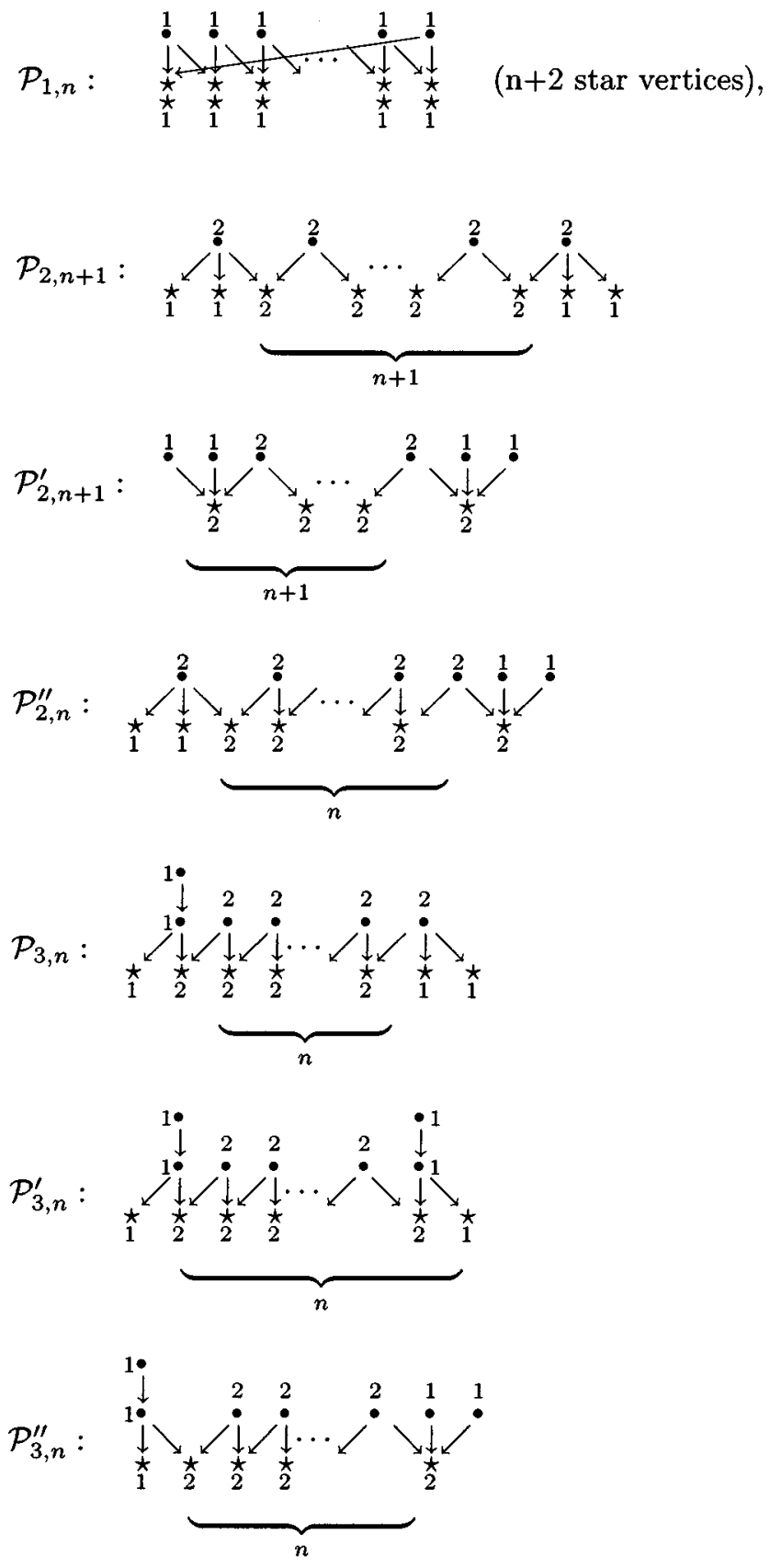

We complete the infinite series $\mathcal{P}_{2, n+1}$ for $n=-1$ and $\mathcal{P}_{3, n}^{\prime}$ for $n=0$ by the following four-peak and three-peak posets respectively. 
<smiles>[3H][13C]([3H])([3H])[3H]</smiles>

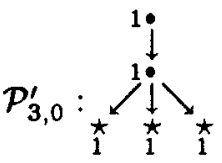

Part 2. One-Peak enlargements $\mathcal{P}_{4}-\mathcal{P}_{8}$ OF Kleiner's posets<smiles>[V]=[W]=[Te]</smiles>

$$
\mathcal{P}_{5}=\mathcal{K}_{2}^{*}:
$$

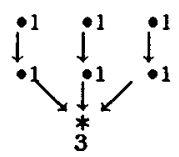

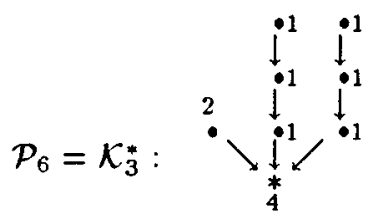

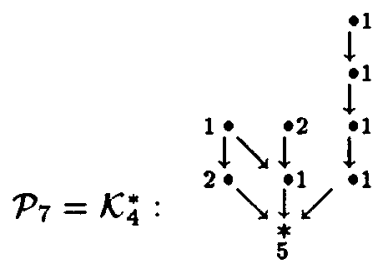

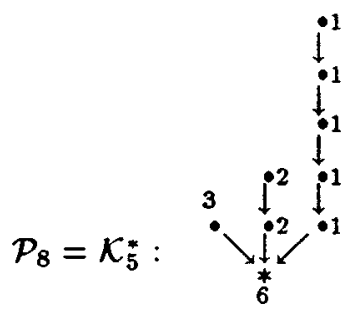

Part 3. TWO-PEAK posets $\mathcal{P}_{9}-\mathcal{P}_{31}$.
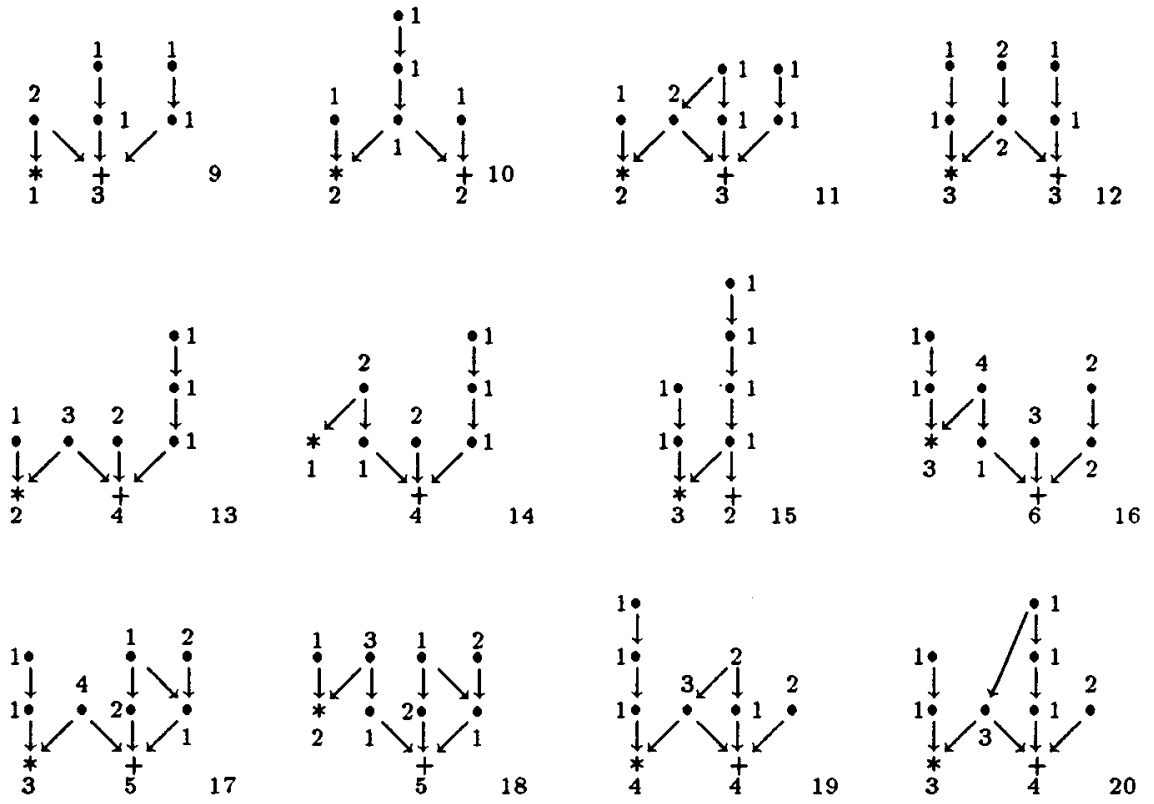

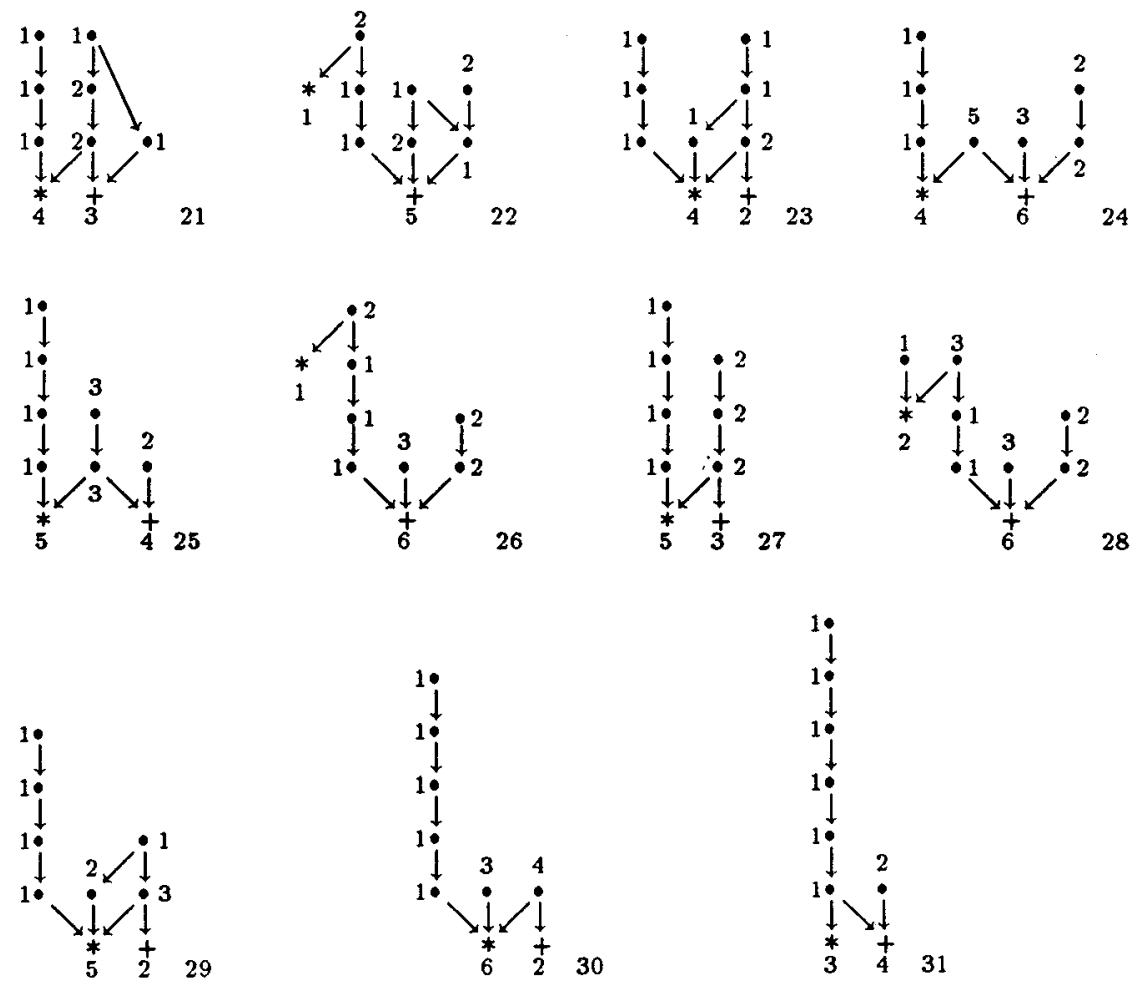

Part 4. Posets $\mathcal{P}_{32}, \ldots, \mathcal{P}_{110}$ With $3 \leq\left|\max \mathcal{P}_{j}\right| \leq 5$
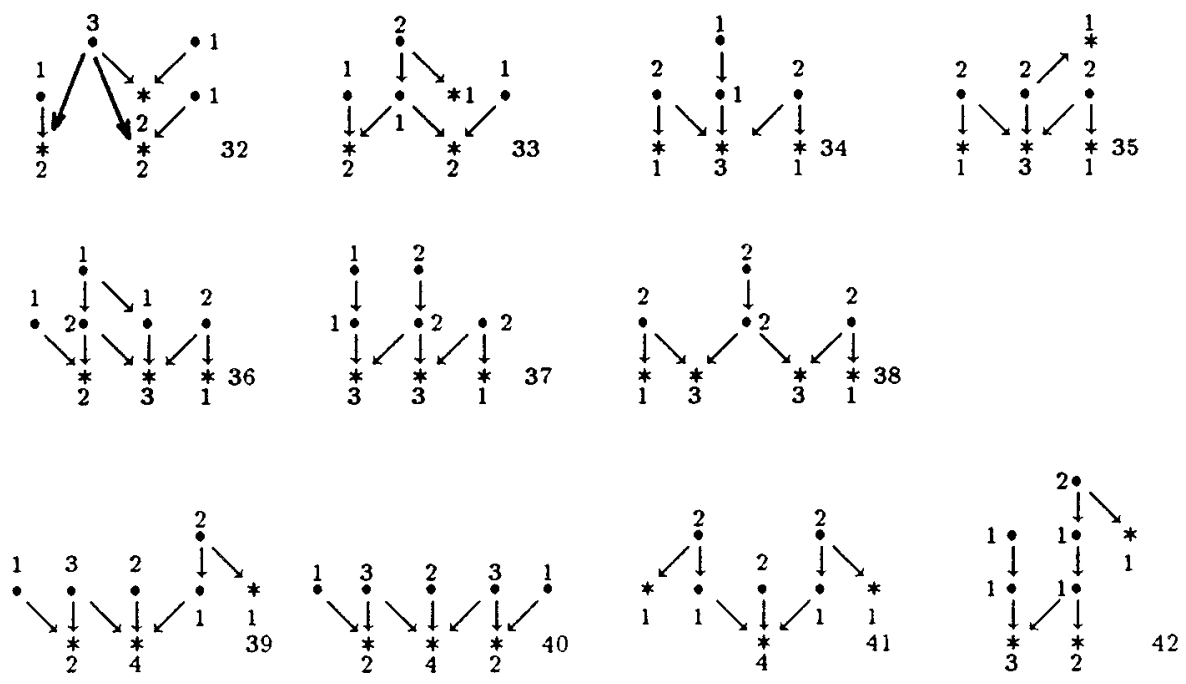

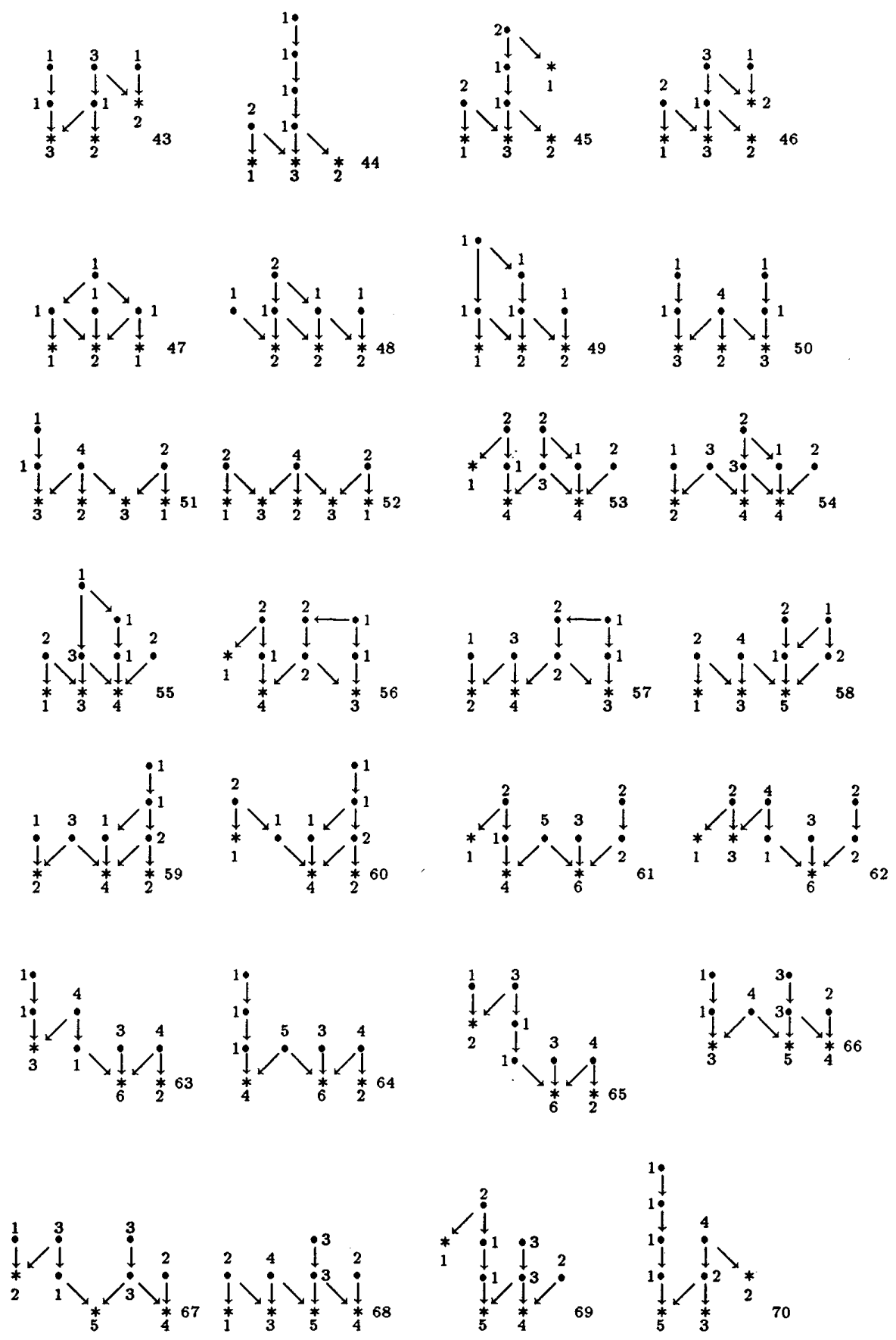

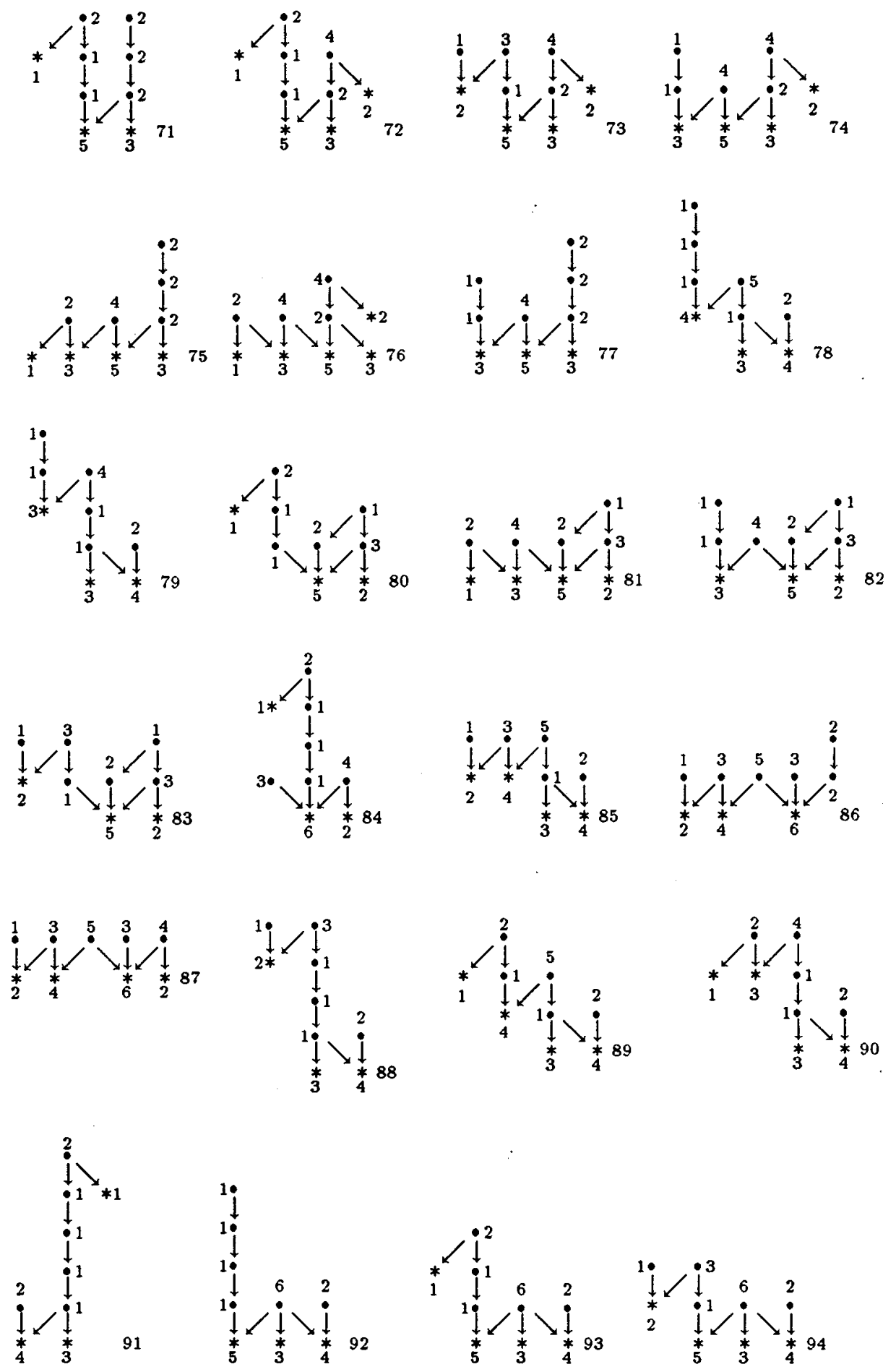

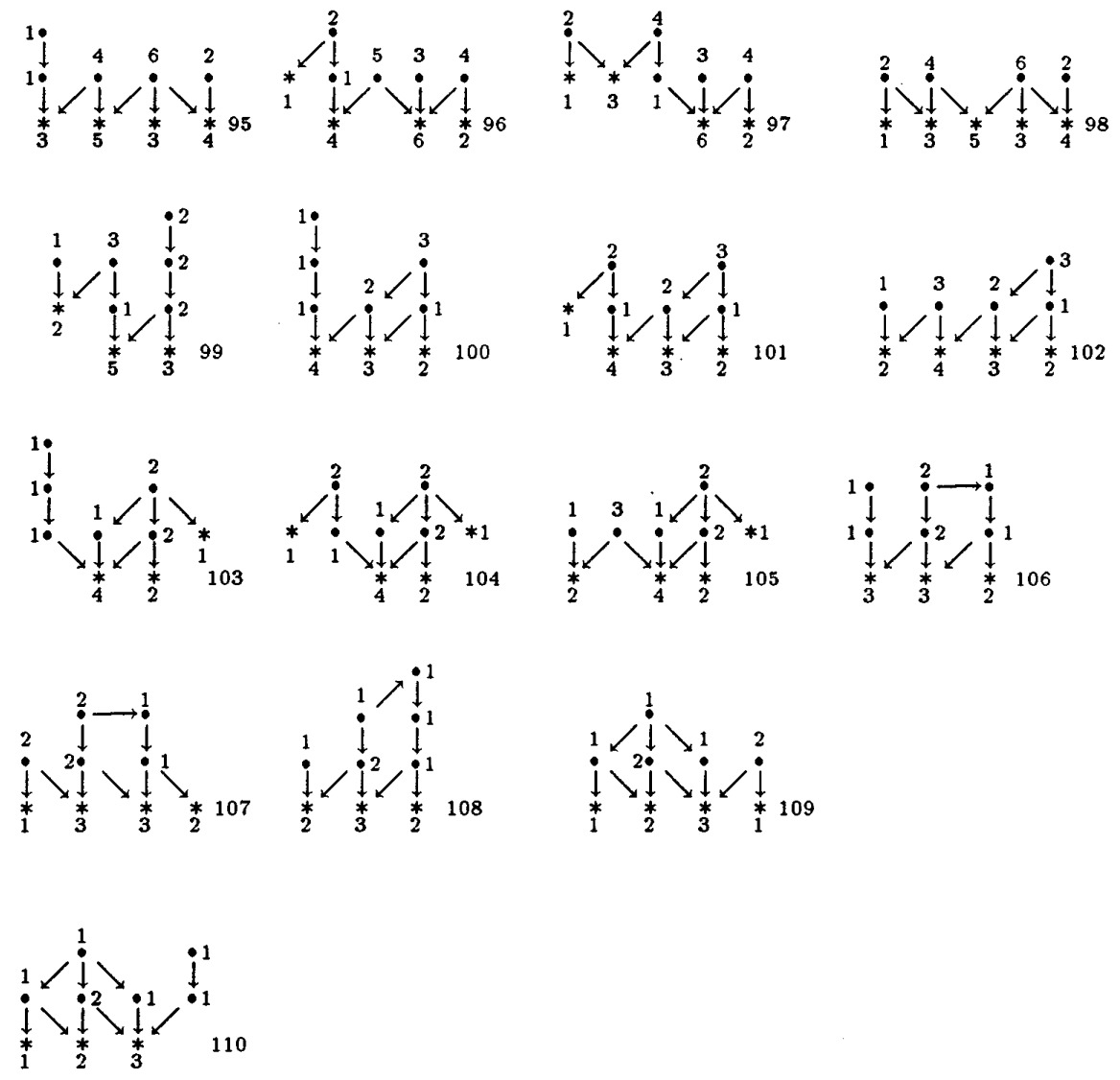

\section{REFERENCES}

1. F. W. Anderson and K. R. Fuller, Rings and Categories of Modules, Graduate Texts in Mathematics 13, Springer, 1974. MR 54:5281

2. D. Arnold, Representations of partially ordered sets and abelian groups, pp. 91-109 in "Abelian Group Theory," ed. L. Fuchs, R. Göbel and P. Schultz, Contemp. Math., Vol. 87, Amer. Math. Soc., Providence, R.I., 1988. MR 90j:20118

3. _ Coxeter correspondences and invariants for representations of finite posets, Manuscript.

4. D. Arnold and F. Richman, Field-independent representations of partially ordered sets, Forum Math. 4 (1992), 349-357. MR 93h:16016

5. W. Baur, On the elementary theory of quadruples of vector spaces, Ann. Math. Logic 19 (1980), 243-357. MR 82g:03056

6. C. Böttinger and R.Göbel, Endomorphism algebras of modules with distinguished partially ordered submodules over commutative rings, Journ. Pure and Appl. Algebra 76 (1991) 121141. MR 93c: 16027

7. _ Modules with distinguished submodules, pp. 97-104 in "Abelian Groups", ed. L. Fuchs, R. Göbel, Marcel Dekker, New York 1993. MR 94c:16042

8. S. Brenner, Endomorphism algebras of vector spaces with distinguished sets of subspaces, J. Algebra, 6 (1967), 100-114. MR 30:4794

9. 127-141. MR 50:13159

10. _ On four subspaces of a vector space, J. Algebra 29 (1974), 587-599. MR 49:7271

11. S. Brenner and M. C. R. Butler, Endomorphism rings of vector spaces and torsion free abelian groups, J. London Math. Soc., 40 (1965), 183-187. MR 30:4794 
12. S. Brenner and C. M. Ringel, Pathological modules over tame rings, J. London Math. Soc. (2) 14 (1976), 207-215. MR 55:5697

13. M.C.R. Butler, Some almost split sequences in torsion-free abelian group theory, pp. 291-302 in "Abelian Group Theory" Gordon and Breach, London 1987. MR 90f:20079

14. A. L. S. Corner, Endomorphism algebras of large modules with distinguished submodules, J. Algebra, 11 (1969), 155-185. MR 38:5838

15. _ Fully rigid systems of modules, Rend. Sem. Mat. Univ. Padova, 82 (1989), 55-66. MR 91d:13016

16. V. Dlab and C. M. Ringel, Indecomposable representations of graphs and algebras, Mem. Amer. Math. Soc. 173, AMS Providence, 1976. MR 56:5657

17. Y. A. Drozd, Coxeter transformations and representations of partially ordered sets, Funct. Anal. and Appl., 8 (1974), 219-225. MR 50:4412

18. M. Dugas and B. Thomé, Countable Butler groups and vector spaces with four distinguished subspaces, J. Algebra 138 (1991), 249-272. MR 92d:20081

19. M. Dugas, R. Göbel and W. May, Free modules with two distinguished submodules, Comm. Algebra, to appear.

20. M. Dugas and R. Göbel, Automorphism groups of fields, Manuscripta Mathematica 85 (1994), 227-242. MR 95k:12006

21. B. Franzen and R. Göbel, The Brenner-Butler-Corner-Theorem and its applications to modules, pp. 209-227 in: "Abelian group theory", ed. R. Göbel and E. A. Walker, Gordon and Breach (1987). MR 83a:20093

22. L. Fuchs, Infinite Abelian Groups, Vol. 1 and Vol. 2, Academic Press, New York 1970, 1973. MR 41:333; MR 50:2362

23. _ L L L inge indecomposable modules in torsion theories, Aequationes Math. 34 (1987), 106-111. MR 88k:13007

24. P. Gabriel, Représentations indécomposables des ensembles ordonnés, Séminaire P. Dubreil, Paris (19722-1973), 1301-1304. MR 55:12581

25. _ Unzerlegbare Darstellungen I, Manuscripta Math. 6 (1972), 71-103. MR 48:11212

26. I I I _ I decomposable representations II, Symposia Math. 11 (1973), 81-104. MR 49:5132

27. I. M. Gelfand and V. A. Ponomarev, Problems of linear algebra and classification of quadruples of subspaces in a finite-dimensional vector space, in: Hilbert Space operators, Tihany 1970, Colloq. Math. János Bolyai, Vol. 5 (North-Holland, Amsterdam-New York), 1972, pp. 163237. MR 50:9896

28. R. Göbel, Vector spaces with five distinguished subspaces, Resultate Math., 11 (1987), 211228. MR 88g:13010

29. R. Göbel and W. May, Four submodules suffice for realizing algebras over commutative rings, J. Pure and Appl. Algebra 65 (1990), 29-43. MR 91j:16036

30. R. Göbel, Modules with distinguished submodules and their endomorphism algebras, pp. 55-64 in "Abelian Groups", ed. L. Fuchs, R. Göbel, Marcel Dekker, New York 1993. MR 93m:20075

31. R. Göbel and M. Ziegler, Very decomposable abelian groups, Math. Z. 200 (1989), 485-496. MR 90e: 20052

32. H.J. v. Höhne, On weakly positive unit forms, Comment. Math. Helv. 63 (1988), 312-336.

33. S. Kasjan and D. Simson, A peak reduction functor for socle projective representations, J. Algebra 187 (1997), 49-70. CMP 97:06

34. O. Kerner, Partially ordered sets of finite representation type, Comm. Algebra 9 (8) (1981), 783-809. MR 82j:06006

35. M. M. Kleiner, Partially ordered sets of finite type, J. Soviet Math. 3 (1975), 607-615. MR 48:06001

36. On the exact representations of partially ordered sets of finite type, J. Soviet Math. 3 (1975), 616-628. MR 48:10912

37. W. May, Endomorphisms of valuated torsionfree modules, pp. 201-207 in "Abelian Groups", ed. L. Fuchs, R. Göbel, Marcel Dekker, New York 1993. MR 93m:20075

38. R. Mines, C. Vinsonhaler and W. J. Wickless, Representations and duality, pp. 295-303 in "Abelian Group Theory and Related Topics", ed. R. Göbel, P. Hill and W. Liebert, Contemp. Math., Vol. 171, Amer. Math. Soc., Providence R.I., 1994. MR 95j:16018

39. B. Mitchell, Theory of categories, Academic Press, New York 1965. MR 34:2647

40. L. A. Nazarova, Partially ordered sets of infinite type, Izv. Akad. Nauk SSR, Ser. Mat., 39 (1975), 963-991 = Math. USSR Izv., 9 (1975), 911-938. MR 53:10664 
41. L. A. Nazarova and A. V. Roiter, Representations of partially ordered sets, J. Soviet. Math. 3 (1975), 585-607.

42. _ Representations of bipartite completed posets, Comment. Math. Helvetici 63 (1988), 498-526. MR 89m:06003

43. F. Richman and E. Walker, Ext in pre-Abelian categories, Pacific Journ. Math. 71 (1977), 521-535. MR 56:3091

44. _ Valuated Groups, Journal of Algebra 56 (1979), 145-167. MR 80k:20053

45. C. M. Ringel, Tame Algebras (On algorithms for solving vector space problems II), pp. 137-287 in Representation Theory I, Lecture Notes in Math., Vol. 831, Springer, 1980. MR 81j:16056

46. _ Infinite-dimensional representations of finite dimensional hereditary algebras, Symposia Math. 23 (1979), 321-412. MR 81i:16032

47. , Tame Algebras and Integral Quadratic Forms, Lecture Notes in Math., Vol. 1099, Springer, 1984. MR 87f:16027

48. C. M. Ringel and H. Tachikawa, QF-3 rings, J. Reine Angew. Math. 272 (1975), 49-72.

49. S. Shelah, Infinite abelian groups, Whitehead problem and some constructions, Israel J. Math. 18 (1974), 243-256. MR 50:9582

50. Classification of first order theories which have structure theory, Bull. Amer. Math. Soc. 12 (1985), 227-232. MR 86h:03072

51. D. Simson, Functor categories in which every flat object is projective, Bull. Acad. Pol. Ser. Math. 22 (1974), 375-380. MR 50:2307

52. Math. 46 (1977), 91-116. MR 58:845

53. _ Linear representations of partially ordered sets and vector space categories, Algebra, Logic and Applications, 4, Gordon and Breach, London 1992. MR 95g:16013

54. _ Posets of finite prinjective type and a class of orders, Journal Pure and Appl. Algebra 90 (1993), 73-103. MR 95b:16011

55. A. G. Zavadski(j) and L. A. Nazarova, Partially ordered sets of finite growth, Func. Anal. and Appl. 16 (1982), 135-137. MR 83k:16022

Fachbereich 6, Mathematik und Informatik, Universität Essen, Universitätsstr. 3, 45117 Essen, Germany

E-mail address: R.Goebel@uni-essen.de

Department of Mathematics, University of Arizona, Tucson, Arizona 85721

E-mail address: may@math.arizona.edu 